\title{
STUDY OF NICOTIANA TABACUM L EXTRACTION, BY METHODS OF LIQUID AND SUPERCRITICAL FLUID EXTRACTION
}

\author{
Yerbol Saginovich Ikhsanov*, Michael Nauryzbaev, Altynay Musabekova, Mereke Alimzhanova, \\ Erlan Burashev \\ Al-Farabi Kazakh National University
}

The article presents a comparative analysis of various methods and conditions of extraction from the plant Nicotiana tabacum I, harvested in the Almaty region of the Republic of Kazakhstan in 2018. The study of extracts obtained by the traditional method (Extraction using non-polar organic solvents at atmospheric pressure) of solvent extraction in particular hexane, and the method of supercritical fluid extraction, carbon dioxide, under various conditions of the process. Three different supercritical extraction modes were selected, namely: at a relatively low pressure of $120 \mathrm{bar}$ and a low temperature of $28^{\circ} \mathrm{C}$, at an elevated temperature with the same pressure $\left(60^{\circ} \mathrm{C}, 120 \mathrm{bar}\right)$ and at an elevated temperature and pressure $\left(80^{\circ} \mathrm{C}, 170 \mathrm{bar}\right)$. As a result of studying the obtained extracts by GC-MS, their qualitative and quantitative composition was identified. It has been established that the largest number of various compounds is extracted by supercritical fluid extraction at a relatively low pressure of 150 bar for this method and a low temperature of $28^{\circ} \mathrm{C}$, but the most popular compound in the studied raw material, nicotine, is extracted with approximately the efficiency similar to the classical method. To increase the yield of the target component, the extraction conditions were changed. As a result, a significant increase in the degree of extraction of the target product was achieved under the following conditions: pressure 120 bar, temperature $60^{\circ} \mathrm{C}$. Under these conditions, the degree of extraction was $47.40 \%$. Conducted to control and more fully study the laws of the extraction process (at elevated pressure) showed less efficiency, only $12.29 \%$. This result is lower in efficiency than the classical extraction method. From the data obtained, it follows that for the extraction of nicotine, a substance belonging to the class of alkaloids and which is resistant to thermal destruction, preferred and most effective, is the mode of supercritical extraction at a temperature of $60^{\circ} \mathrm{C}$ and a pressure of 120 bar.

Key words: Nicotiana tabacum I, liquid extraction, supercritical fluid extraction, GC-MS, nicotine

\section{INTRODUCTION}

It is known that the composition of plant extracts directly depends on many factors, such as the temperature of the extraction process, the extraction time, the ratio of plant raw material / extractant and, of course, the main factor is the nature of the solvent or solvent system used.

Depending on the tasks to be solved, a rich arsenal of organic solvents such as hexane, chloroform or benzene is used to extract biologically active substances.

However, most organic solvents are highly toxic compounds; exhibit an accumulation effect, which requires the introduction of additional stages of cleaning the drug substance and additional methods of quality control of such preparations. These measures lead to an inevitable rise in price of the final product. In addition, a significant part of organic solvents are in one way or another petrochemical products, their price will inevitably increase in the future.

In this regard, the last decades are actively studying and developing new methods for extracting complexes of biologically active substances from plant materials.

Supercritical extraction methods turned out to be very effective and have a number of advantages.
Supercritical fluids have been investigated since the last century, and at first the use of supercritical toluene in the processing of shale oil in the 1970s was of the greatest commercial interest. Supercritical water is also being investigated as a means of destroying toxic waste and as an exotic synthesis environment.

In the field of chemistry in the processing of plant materials, supercritical carbon dioxide, the critical temperature of which is $31^{\circ} \mathrm{C}$, is of the greatest interest. Biological materials can be processed at $35^{\circ} \mathrm{C}$, which contributes to their safety from thermal destruction. The density of supercritical $\mathrm{CO}_{2}$ at a pressure of about 200 bar is similar in efficiency to hexane, and the solvation characteristics during extraction are also similar to hexane; thus, it acts as a non-polar solvent. [1]

The main advantage is that a slight decrease in temperature, or a slightly greater decrease in pressure, will lead to an almost complete precipitation of the target component.

Moreover, under normal conditions, carbon dioxide is a gas, so after the extraction process is completed; it becomes possible to obtain extracts with no solvent at all.

Examples of industrial products obtained using supercritical technologies include decaffeinated coffee, 
cholesterol-free oil, lean meat, rose oil, etc. The solvation characteristics of supercritical $\mathrm{CO}_{2}$ can be modified by adding a co-solvent such as ethanol, which can significantly increase the efficiency of extraction, however, the solvent residue in the product somewhat levels the main advantage of the process, which consists in the complete absence of impurities in the final extract [2,3].

As a plant raw material, we chose the plant Nicotiana tabacum I, due to the high content of biologically active substances in tobacco leaves, in particular, saturated and unsaturated fatty acids, fat-soluble vitamins, waxes, terpenes, terpenoids, pigments, alkaloids, essential oils and phytosterols, showing growth-regulating, fungicidal and insecticidal activity [4-15] .

It should also be noted that due to a drop in demand for tobacco products, there is a release of a large amount of high-quality plant materials.

For comparison, two extraction methods were chosen, classical liquid extraction with hexane and supercritical extraction with carbon dioxide.

Objective: study of the qualitative and quantitative composition of the obtained extracts.

\section{Materials and methods}

The classical method of extraction (Extraction using non-polar organic solvents at atmospheric pressure) was carried out with hexane in the ratio of raw materials $\backslash$ extractant 1:10 with infusion for 72 hours at a temperature of $28^{\circ} \mathrm{C}$.

The supercritical extraction method was carried out on a Thar SFE-1000 $\mathrm{CO}_{2}$-extraction unit under the following conditions: the temperature range in the reactor is 28$80^{\circ} \mathrm{C}$, the pressure of $\mathrm{CO}_{2}$ is $120-170$ bar.

The obtained extract was investigated by gas chromatography on an Agilent Technologies 7890N / 5973N GC / MS gas chromatograph with a mass selective detector under the following conditions: a DB-35MS column (30 $\mathrm{mx} 250 \mathrm{~mm} \times 0.25 \mathrm{~mm}$ ) was used, the helium carrier gas velocity $1 \mathrm{ml} / \mathrm{min}$ The chromatographic temperature is programmed from $40^{\circ} \mathrm{C}$ (holding $0 \mathrm{~min}$ ) to $300^{\circ} \mathrm{C}$ with a heating rate of $5^{\circ} \mathrm{C} / \mathrm{min}$ (holding $5 \mathrm{~min}$ ). Detection is carried out in the SCAN m / z mode 34-800. The software Agilent MSD ChemStation (version 1701EA) was used to control the gas chromatography system, record and process the obtained results and data. Data processing included determination of retention times, peak areas, as well as processing of spectral information obtained using a mass spectrometric detector. To decode the mass spectra obtained, the libraries Wiley 7 th edition and NIST'02 were used (the total number of spectra in the libraries is more than 550 thousand) $[16,17]$.

\section{Results and discussion}

In the course of the work, 7 extracts were obtained under various methods and conditions of extraction.

The data presented in tables 1-7
Table 1: The results of the study of hexane extract of the plant Nicotiana tabacum I by the method of chromatography-mass spectrometry

\begin{tabular}{|c|c|c|c|}
\hline № & $\begin{array}{l}\text { Retention } \\
\text { time, min }\end{array}$ & Name of compounds & $\begin{array}{c}\text { Content } \\
\%\end{array}$ \\
\hline 1 & 7,84 & Sorbicacid & 8,80 \\
\hline 2 & 9,54 & Benzoicacid & 12,22 \\
\hline 3 & 10,91 & Tetradecane & 0,73 \\
\hline 4 & 11,54 & Heptadecane, 4-methyl & 0,14 \\
\hline 5 & 11,81 & $\begin{array}{l}\text { 6,8-nonadien-2-one, } \\
\text { 8-methyl-5- (1-methyleth- } \\
\text { yl) - (E) }\end{array}$ & 1,00 \\
\hline 6 & 12,23 & Pentadecane & 1,04 \\
\hline 7 & 12,44 & Nicotine & 19,52 \\
\hline 8 & 12,91 & 1-dodecanol & 0,27 \\
\hline 9 & 13,09 & $\begin{array}{l}\text { 5,9-indian-2-one, 6,10-di- } \\
\text { methyl }\end{array}$ & 0,35 \\
\hline 10 & 13,49 & Hexadecane & 1,20 \\
\hline 11 & 13,88 & $\begin{array}{l}\text { Quinoline, 1,2-dihy- } \\
\text { dro-2,2,4-trimethyl }\end{array}$ & 0,29 \\
\hline 12 & 14,49 & $\begin{array}{l}\text { Pentadecane, } \\
2,6,10,14 \text {-tetramethyl }\end{array}$ & 0,39 \\
\hline 13 & 14,68 & Heptadecane & 1,08 \\
\hline 14 & 15,45 & Megastigmasteron & 0,83 \\
\hline 15 & 15,70 & $\begin{array}{l}2(4 \mathrm{H}) \text {-benzofura- } \\
\text { none, 5,6,7,7a-tetrahy- } \\
\text { dro-4,4,7a-trimethyl-, }(\mathrm{R})\end{array}$ & 0,87 \\
\hline 16 & 15,81 & Octadecane & 1,23 \\
\hline 17 & 16,07 & 3-hydroxy- $\beta$-damascon & 0,64 \\
\hline 18 & 16,45 & $\begin{array}{l}\text { 3,7,11,15-tetrameth- } \\
\text { yl-2-hexadecen-1-ol }\end{array}$ & 2,89 \\
\hline 19 & 16,51 & $\begin{array}{l}\text { 2-cyclohexen-1-one, } \\
\text { 4- (3-hydroxy-1-butenyl) } \\
\text {-3,5,5-trimethyl- }\end{array}$ & 2,03 \\
\hline 20 & 17,00 & Nonadecane & 1,49 \\
\hline 21 & 17,45 & $\begin{array}{l}\text { 2-cyclohexen-1-one, } \\
\text { 4- (3-hydroxybutyl) } \\
\text {-3,5,5-trimethyl }\end{array}$ & 0,53 \\
\hline 22 & 18,07 & $\begin{array}{l}\text { Bicyclo [3.1.1] hept-2- } \\
\text { ene, 2,2 '- (1,2-ethanedi- } \\
\text { yl) bis [6,6-dimethyl] }\end{array}$ & 0,26 \\
\hline 23 & 18,48 & Heptadecane, 9-hexyl- & 0,46 \\
\hline 24 & 18,58 & $\begin{array}{l}\text { Hexadecanoicacid, meth- } \\
\text { ylester }\end{array}$ & 0,70 \\
\hline
\end{tabular}




\begin{tabular}{|c|c|c|c|}
\hline 25 & 19,15 & $\begin{array}{l}\text { 7-isopropenyl-1,4a-di- } \\
\text { methyl-4,4a, } \\
\text { 5,6,7,8-hexahy- } \\
\text { dro-3H-naphthalen-2-one }\end{array}$ & 0,36 \\
\hline 26 & 19,50 & n-hexadecanoicacid & 0,73 \\
\hline 27 & 20,36 & $\begin{array}{l}\text { Cyclodecacyclotetrade- } \\
\text { cene, 14,15-didehydro } \\
-1,4,5,8,9,10,11,12, \\
13,16,17,18,19,20- \\
\text { tetradecahydro }\end{array}$ & 2,09 \\
\hline 28 & 23,79 & $\begin{array}{l}\text { 4,8,13-Cyclotetra- } \\
\text { decatriene-1,3-diol, } \\
\text { 1,5,9-trimethyl-12- } \\
\text { (1-methylethyl) }\end{array}$ & 14,49 \\
\hline 29 & 24,71 & Retinol & 13,78 \\
\hline 30 & 26,37 & 1-heptacantanol & 7,17 \\
\hline 31 & 26,59 & 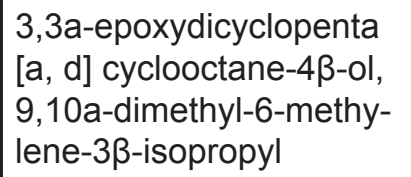 & 2,42 \\
\hline \multicolumn{3}{|c|}{ Sum } & 100,00 \\
\hline
\end{tabular}

From table 1 it can be seen that the hexane extract from the plant Nicotiana tabacum / revealed a large $(19.52 \%)$ nicotine content of the natural alkaloid of the pyridine series, typical of the solanaceous family plants (Solanaceae) to which the object under study belongs. It should be noted that nicotine is often the target component in the processing of tobacco and the effectiveness of the extraction process is determined by its amount and completeness of extraction [18-23].

Found a high content of organic acids such as sorbic acid and benzoic acid, a significant content of hydrocarbons with a total content of $9.38 \%$, a high content of vitamin $A$, retinol $(13.78 \%)$, content of 1-heptatriacontanol $(7.17 \%)$, which is a growth regulating agent.

Table 2: Results of chromatography-mass spectrometric analysis of the extract of the plant Nicotiana tabacum I, obtained by the method of supercritical fluid $\mathrm{CO}_{2}$ extraction under conditions of 150 bar $28^{\circ} \mathrm{C}$

\begin{tabular}{|c|c|l|c|}
\hline № & $\begin{array}{c}\text { Retention } \\
\text { time, min }\end{array}$ & Name of compounds & $\begin{array}{c}\text { Content } \\
\%\end{array}$ \\
\hline 1 & 5,22 & 1-butanol & 0,19 \\
\hline 2 & 5,40 & Benzene, (1-methylethyl) & 0,20 \\
\hline 3 & 5,58 & Benzene, 1,3-dimethyl & 0,06 \\
\hline 4 & 5,63 & $\begin{array}{l}\text { Propane, 1- (1-methyle- } \\
\text { thoxy) }\end{array}$ & 0,14 \\
\hline 5 & 5,71 & Methanesulfonylchloride & 0,14 \\
\hline 6 & 5,88 & 1-butanol, 3-methyl & 4,39 \\
\hline
\end{tabular}

\begin{tabular}{|c|c|c|c|}
\hline 7 & 6,00 & $\begin{array}{l}\text { Cyclopentane, } \\
\text { 1,2,4-trimethyl }\end{array}$ & 0,05 \\
\hline 8 & 6,05 & Furan, 2-Pentyl & 0,05 \\
\hline 9 & 6,11 & Hexanoicacid, ethylester & 0,07 \\
\hline 10 & 6,61 & $\begin{array}{l}\text { Ethanone, 2- (formyloxy) } \\
\text {-1-phenyl }\end{array}$ & 0,66 \\
\hline 11 & 7,14 & 2-propanone, 1-hydroxy & 0,09 \\
\hline 12 & 7,36 & Pyrazine, 2,5-dimethyl & 0,05 \\
\hline 13 & 7,43 & Pyrazine, 2,6-dimethyl & 0,45 \\
\hline 14 & 7,51 & 5-Hepten-2-one, 6-methyl & 0,04 \\
\hline 15 & 7,63 & 1-hexanol & 0,03 \\
\hline 16 & 8,33 & Ethanol, 2-butoxy & 0,42 \\
\hline 17 & 8,68 & Octanoicacid, ethylester & 0,07 \\
\hline 18 & 8,86 & 1-hexanol, 2-ethyl & 0,30 \\
\hline 19 & 8,91 & Aceticacid & 4,08 \\
\hline 20 & 9,21 & furfural & 0,07 \\
\hline 21 & 9,37 & 1-hexanol, 2-ethyl & 0,16 \\
\hline 22 & 9,70 & Propanoicacid & 0,53 \\
\hline 23 & 9,99 & Nenanoicacid, ethylester & 0,14 \\
\hline 24 & 10,48 & $\begin{array}{l}\text { Methoxyaceticacid, 3-tri- } \\
\text { decyl ether }\end{array}$ & 0,33 \\
\hline 25 & 10,68 & Propyleneglycol & 0,38 \\
\hline 26 & 11,16 & Butaneacid & 0,39 \\
\hline 27 & 11,35 & biturolactone & 0,09 \\
\hline 28 & 11,55 & 2-furanmethanol & 0,10 \\
\hline 29 & 11,79 & \begin{tabular}{|l|} 
Butane acid, 4- (1,1-di- \\
methylethoxy) -3-hy- \\
droxy-, methyl ester, (R) -
\end{tabular} & 0,12 \\
\hline 30 & 11,90 & heptadecane & 0,08 \\
\hline 31 & 12,37 & \begin{tabular}{|l|} 
6,8-nonadien-2-one, \\
8-methyl-5- (1-methyleth- \\
yl) -, (E) \\
\end{tabular} & 0,78 \\
\hline 32 & 12,52 & Oxime-, methoxyphenyl & 0,19 \\
\hline 33 & 12,93 & 1,3-propandiol & 0,08 \\
\hline 34 & 12,99 & $\begin{array}{l}\text { Ethanone, 1- (4-methyl- } \\
\text { phenyl) }\end{array}$ & 0,10 \\
\hline 35 & 13,06 & Pentanoicacid, 3-methyl & 0,68 \\
\hline 36 & 13,23 & $\begin{array}{l}\text { 1,2-propandiol, 3-me- } \\
\text { thoxy }\end{array}$ & 0,94 \\
\hline 37 & 13,60 & Hexanoicacid & 0,32 \\
\hline 38 & 13,76 & $\begin{array}{l}\text { 5,9-undecane-2-one, } \\
\text { 6,10-dimethyl-, (Z) }\end{array}$ & 0,19 \\
\hline 39 & 14,08 & Nicotine & 19,34 \\
\hline
\end{tabular}




\begin{tabular}{|c|c|c|c|}
\hline 40 & 14,43 & $\begin{array}{l}\text { 3,7,11,15-tetrameth- } \\
\text { yl-2-hexadecen-1-ol }\end{array}$ & 2,34 \\
\hline 41 & 14,57 & $\begin{array}{l}\text { Glycolaldehydedimethy- } \\
\text { lacetal }\end{array}$ & 0,21 \\
\hline 42 & 14,70 & $\begin{array}{l}\text { Malonicacid, 2-butyldo- } \\
\text { decyl ester }\end{array}$ & 0,18 \\
\hline 43 & 14,85 & 1-dodecanol & 0,16 \\
\hline 44 & 15,01 & maltol & 0,16 \\
\hline 45 & 15,08 & $\begin{array}{l}\text { Ethanone, 1- (1H-pyrrol- } \\
2 \text {-yl) - }\end{array}$ & 0,24 \\
\hline 46 & 15,16 & Dodecylacrylate & 0,88 \\
\hline 47 & 15,28 & Diethylpimelate & 0,13 \\
\hline 48 & 15,41 & Phenol & 0,20 \\
\hline 49 & 15,72 & $\begin{array}{l}\text { 7-Methyl-Z-tetrade- } \\
\text { cene-1-ol }\end{array}$ & 0,27 \\
\hline 50 & 15,85 & Octanoicacid & 0,37 \\
\hline 51 & 16,25 & $\begin{array}{l}\text { 7-methoxy-2,2,4,8-te- } \\
\text { tramethylcyclo [5.3.1.0 } \\
(4.11)] \text { undecane }\end{array}$ & 0,72 \\
\hline 52 & 16,36 & Diethylsuberate & 0,21 \\
\hline 53 & 16,69 & Sorbicacid & 1,77 \\
\hline 54 & 16,97 & $\begin{array}{l}\text { 10-methyldodec-2-en-4- } \\
\text { olide }\end{array}$ & 0,95 \\
\hline 55 & 17,58 & megastigmasteron & 1,54 \\
\hline 56 & 18,05 & $\begin{array}{l}\text { Hexadecanoicacid, eth- } \\
\text { ylester }\end{array}$ & 0,84 \\
\hline 57 & 18,58 & $\begin{array}{l}\text { 1,2,3-propantriol, 1-ac- } \\
\text { etate }\end{array}$ & 2,44 \\
\hline 58 & 19,43 & Glycerol & 12,27 \\
\hline 59 & 20,03 & $\begin{array}{l}\text { 5,9,13-pentade- } \\
\text { catrien-2-one, } \\
6,10,14 \text {-trimethyl-, (E, } \\
\text { E) - }\end{array}$ & 0,21 \\
\hline 60 & 21,31 & Benzoicacid & 16,67 \\
\hline 61 & 21,90 & $\begin{array}{l}\text { trans-dehydroandroste- } \\
\text { rone, pentafluoropropio- } \\
\text { nate }\end{array}$ & 0,41 \\
\hline 62 & 22,42 & 2,3'-dipyridyl & 0,56 \\
\hline 63 & 22,61 & $\begin{array}{l}\text { transdehydroandroste- } \\
\text { rone, trifluoroacetate }\end{array}$ & 0,44 \\
\hline 64 & 23,38 & $\begin{array}{l}\text { Bicyclo [3.1.1] hept-2- } \\
\text { ene, 2,2 '- (1,2-ethanedi- } \\
\text { yl) bis [6,6-dimethyl- }\end{array}$ & 1,13 \\
\hline 65 & 23,86 & 3-hydroxy- $\beta$-damascon & 0,66 \\
\hline
\end{tabular}

\begin{tabular}{|c|c|c|c|}
\hline 66 & 26,00 & $\begin{array}{l}\text { 4,8,13-Cyclotetrade- } \\
\text { catatriene-1,3-diol, } \\
\text { 1,5,9-trimethyl-12-- } \\
\text { (1-methylethyl) - }\end{array}$ & 3,06 \\
\hline 67 & 27,04 & $\begin{array}{l}\text { 2-cyclohexen-1-one, } \\
\text { 4- (3-hydroxy-1-butenyl) } \\
\text {-3,5,5-trimethyl }\end{array}$ & 3,29 \\
\hline 68 & 29,92 & Tetradecanoicacid & 2,65 \\
\hline 69 & 30,63 & triacontan & 5,79 \\
\hline 70 & 30,90 & $\begin{array}{l}\text { Cyclopenta [a, d] cy- } \\
\text { clooctene-5-one, } \\
\text { 1,2,3,3a, 4,5,6,8,9,9a, } \\
\text { 10,10a-dodecahydro-7- } \\
\text { (1-methylethyl) -1,9a- di- } \\
\text { methyl-4-methylene }\end{array}$ & 2,76 \\
\hline \multicolumn{3}{|c|}{ Sum } & 100,00 \\
\hline
\end{tabular}

In the supercritical extract, a high content of organic acids, in particular, sorbic acid and benzoic acid, is noted; a greater number of compounds are also isolated. 31 compounds were identified in the hexane extract, while in the carbon-dioxide supercritical - 70 compounds. The content of the main product of extraction, namely nicotine, was $19.34 \%$, which is almost identical to the content in the extract obtained by the traditional method of solvent extraction [24-29].

To increase the yield of nicotine, the conditions for supercritical extraction were changed, namely: the process temperature was increased from 28 to $60^{\circ} \mathrm{C}$.

The results of the study, obtained under these conditions, the extract is presented in table 3 .

Table 3: Results of chromato-mass spectrometric analysis of the extract of the plant Nicotiana tabacum I obtained by the method of supercritical fluid $\mathrm{CO}_{2}$ extraction under conditions of 150 bar $60^{\circ} \mathrm{C}$

\begin{tabular}{|c|c|l|c|}
\hline № & $\begin{array}{c}\text { Retention } \\
\text { time, } \min \end{array}$ & \multicolumn{1}{|c|}{ Name of compounds } & $\begin{array}{c}\text { Content } \\
\%\end{array}$ \\
\hline 1 & 6,334 & $\begin{array}{l}\text { 2,4-hexadienoic acid, } \\
\text { methylester }\end{array}$ & 0,21 \\
\hline 2 & 6,99 & Benzylalcohol & 0,37 \\
\hline 3 & 10,829 & $\begin{array}{l}\text { 6,8-nonadien-2-one, } \\
\text { 8-methyl-5- (1-methyleth- } \\
\text { yl)-, (E) - }\end{array}$ & 0,99 \\
\hline $\mathbf{4}$ & $\mathbf{1 1 , 9 4 2}$ & Nicotine & $\mathbf{2 3 , 7 0}$ \\
\hline 5 & 12,09 & $\begin{array}{l}\text { 2,2-dimethyl-1- (3-oxo- } \\
\text { but-1-enyl) -cyclopentan- } \\
\text { ecarboxydehyde }\end{array}$ & 1,13 \\
\hline 6 & 13,249 & $\begin{array}{l}\text { Cyclohexanone, 2,3-di- } \\
\text { methyl-2- (3-oxobutyl) }\end{array}$ & 0,58 \\
\hline 7 & 13,392 & $\begin{array}{l}\text { Pyridine, 3- (3,4-dihydro- } \\
\text { 2H-pyrrol-5-yl) - }\end{array}$ & 0,40 \\
\hline
\end{tabular}




\begin{tabular}{|c|c|c|c|}
\hline 8 & 13,596 & heptadecane & 0,17 \\
\hline 9 & 13,999 & Diethylsuberate & 0,52 \\
\hline 10 & 14,209 & $\begin{array}{l}\text { Tricyclo [6.3.0.0 (1.5)] } \\
\text { undec-2-en-4-one, 5,9-di- } \\
\text { methyl- }\end{array}$ & 0,38 \\
\hline 11 & 14,417 & megastigmasteron & 0,94 \\
\hline 12 & 14,695 & $\begin{array}{l}2(4 \mathrm{H}) \text {-benzofuranone, } \\
\text { 5,6,7,7a-tetrahydro- } \\
\text { 4,4,7a-trimethyl-, }(\mathrm{R}) \text { - }\end{array}$ & 1,15 \\
\hline 13 & 14,851 & 2,3'-dipyridyl & 0,35 \\
\hline 14 & 15,039 & 3-hydroxy- $\beta$-damascon & 0,81 \\
\hline 15 & 15,335 & Fitol, acetate & 2,81 \\
\hline 16 & 15,525 & $\begin{array}{l}\text { 2-cyclohexen-1-one, } \\
\text { 4- (3-hydroxy-1-butenyl) } \\
\text {-3,5,5-trimethyl- }\end{array}$ & 4,47 \\
\hline 17 & 16,228 & \begin{tabular}{|l} 
] 2-Cyclohexen-1-one, \\
4- (3-hydroxybutyl) \\
-3,5,5-trimethyl- \\
\end{tabular} & 1,22 \\
\hline 18 & 16,653 & DL-xylitol, 1-benzoate & 2,85 \\
\hline 19 & 16,867 & $\begin{array}{l}\text { Hexadecanoicacid, meth- } \\
\text { ylester }\end{array}$ & 0,53 \\
\hline 20 & 17,484 & $\begin{array}{l}\text { Hexadecanoicacid, ethy- } \\
\text { lester }\end{array}$ & 3,09 \\
\hline 21 & 17,579 & n-hexadecanoicacid & 3,54 \\
\hline 22 & 18,301 & $\begin{array}{l}\text { Bicyclo [3.1.1] hept-2-ene, } \\
2,2 \text { '- (1,2-ethanediyl) bis } \\
\text { [6,6-dimethyl- }\end{array}$ & 0,96 \\
\hline 23 & 18,573 & Dibutylphthalate & 0,91 \\
\hline 24 & 18,959 & $\begin{array}{l}\text { 4,8,13-Cyclotetrade- } \\
\text { catatriene-1,3-diol, } \\
\text { 1,5,9-trimethyl-12- } \\
\text { (1-methylethyl) - }\end{array}$ & 2,47 \\
\hline 25 & 19,327 & $\begin{array}{l}\text { 2-pentenoic } \\
\text { acid, 5- (decahy- } \\
\text { dro-5,5,8a-trimeth- } \\
\text { yl-2-methylene-1-naph- } \\
\text { thalenyl) -3-methyl }\end{array}$ & 5,45 \\
\hline 26 & 19,728 & $\begin{array}{l}\text { Cyclopenta [a, d] cyclooc- } \\
\text { tene-5-one, 1,2,3,3a, } \\
4,5,6,8,9,9 a, 10,10 a-d o- \\
\text { decahydro-7- (1-meth- } \\
\text { ylethyl) -1,9a- dimeth- } \\
\text { yl-4-methylene }\end{array}$ & 3,25 \\
\hline 27 & 19,774 & metasterone & 0,98 \\
\hline 28 & 20,575 & $\begin{array}{l}\text { 2-pentenoic } \\
\text { acid, 5- (decahy- } \\
\text { dro-5,5,8a-trimeth- } \\
\text { yl-2-methylene-1-naph- } \\
\text { thalenyl) -3-methyl }\end{array}$ & 1,20 \\
\hline
\end{tabular}

\begin{tabular}{|c|c|c|c|}
\hline 29 & 21,185 & $\begin{array}{l}\text { 4,8,13-Cyclotetrade- } \\
\text { catatriene-1,3-diol, } \\
\text { 1,5,9-trimethyl-12- } \\
\text { (1-methylethyl) - }\end{array}$ & 5,44 \\
\hline 30 & 22,984 & heptacosan & 4,97 \\
\hline 31 & 23,114 & Glycerin 1-palmitate & 3,75 \\
\hline 32 & 23,673 & Octacosan & 1,12 \\
\hline 33 & 24,083 & Hexacosan, 9 octyl & 0,85 \\
\hline 34 & 24,443 & nonacosan & 4,05 \\
\hline 35 & 25,097 & triacontan & 0,96 \\
\hline 36 & 25,903 & gentriacontan & 5,40 \\
\hline 37 & 26,697 & dotriocontan & 1,81 \\
\hline 38 & 27,223 & hexatriacontan & 1,69 \\
\hline 39 & 27,691 & tritriacontan & 3,08 \\
\hline 40 & 28,041 & 28-Nor-17ß $(\mathrm{H})$-gopan & 0,43 \\
\hline 41 & 28,47 & $\begin{array}{l}\text { Tetracosane, 12-decyl-12- } \\
\text { nonyl }\end{array}$ & 0,42 \\
\hline 42 & 29,009 & $\begin{array}{l}\text { 17. Alpha. } 21 \beta-28,30-\text { bis- } \\
\text { norpan }\end{array}$ & 0,60 \\
\hline \multicolumn{3}{|c|}{$\overline{\text { Sum }}$} & 100,00 \\
\hline
\end{tabular}

In the data presented in Table 3, there is a significant increase in the efficiency of nicotine extraction of $23.70 \%$, while in previous processes the yield was less than $20 \%$. Compared with supercritical extraction at low temperatures, in this case there is a smaller number of identified compounds - 42, against 70 , besides a large amount of compounds belonging to groups of alcohols and ethers were found in the extract obtained in the process with heating besides nicotine.

To determine the optimal mode of extraction, an extraction process was carried out at elevated pressure and temperature, namely: the extraction conditions of $60^{\circ} \mathrm{C}$ were established at a pressure of 170 bar, the data obtained after analyzing the total extract are presented in Table 4.

Table 4: Results of chromatography-mass spectrometric analysis of the extract of the plant Nicotiana tabacum I obtained by the method of supercritical fluid $\mathrm{CO}_{2}$ extraction under conditions of 170 bar $60^{\circ} \mathrm{C}$

\begin{tabular}{|c|c|l|c|}
\hline № & $\begin{array}{c}\text { Retention } \\
\text { time, } \min \end{array}$ & Name of compounds & $\begin{array}{c}\text { Content } \\
\%\end{array}$ \\
\hline 1 & 6,25 & Aceticacidbutylester & 14,36 \\
\hline 2 & 8,29 & ethylbenzene & 1,78 \\
\hline 3 & 14,84 & Sorbicacid & 17,56 \\
\hline 4 & 16,57 & Benzoicacid & 28,81 \\
\hline 5 & 18,55 & $\begin{array}{l}\text { 1-isopropyl-4-meth- } \\
\text { yl-1,4-cyclohexadiene }\end{array}$ & 0,46 \\
\hline
\end{tabular}




\begin{tabular}{|c|c|c|c|}
\hline 6 & 19,20 & Nicotine & 12,29 \\
\hline 7 & 22,18 & Styron & 0,99 \\
\hline 8 & 23,14 & 9 octadecen & 2,44 \\
\hline 9 & 23,21 & $\begin{array}{l}\text { 2-cyclohexen-1-one, } \\
\text { 4- (3-hydroxy-1-butenyl) } \\
\text {-3,5,5-trimethyl-, [R- [R *, } \\
\left.R^{*}-(E)\right]\end{array}$ & 0,82 \\
\hline 10 & 24,68 & $\begin{array}{l}\text { Hexadecanoicacidmeth- } \\
\text { ylester }\end{array}$ & 0,58 \\
\hline 11 & 26,37 & Dibutylphthalate & 0,50 \\
\hline 12 & 26,67 & diethylbenzene & 0,72 \\
\hline 13 & 26,73 & $\begin{array}{l}\text { 4,8,13-Cyclotetrade- } \\
\text { catatriene-1,3-diol, } \\
\text { 1,5,9-trimethyl-12- } \\
\text { (1-methylethyl) - }\end{array}$ & 0,71 \\
\hline 14 & 26,84 & $\begin{array}{l}\text { Methyl 8,11,14-heptade- } \\
\text { cathoenoate }\end{array}$ & 0,76 \\
\hline 15 & 26,91 & $\begin{array}{l}\text { Cycloheptane, 4-methy- } \\
\text { lene-1-methyl-2- (2-meth- } \\
\text { yl-1-propen-1-yl) -1-vinyl- }\end{array}$ & 1,40 \\
\hline 16 & 27,08 & \begin{tabular}{|l} 
Cyclohexanethanol, \\
4-methyl- $\beta$-methylene- \\
\end{tabular} & 2,92 \\
\hline 17 & 27,16 & $\begin{array}{l}\text { 4,8,13-Cyclotetrade- } \\
\text { catatriene-1,3-diol, } \\
\text { 1,5,9-trimethyl-12- } \\
\text { (1-methylethyl) - }\end{array}$ & 1,07 \\
\hline 18 & 27,48 & $\begin{array}{l}(\mathrm{R}) \text { - (-) - 14-methyl-8- } \\
\text { hexadecen-1-ol }\end{array}$ & 2,01 \\
\hline 19 & 27,53 & $\begin{array}{l}\text { Ethanol, 1- (1-cyclohex- } \\
\text { enyl) }\end{array}$ & 0,81 \\
\hline 20 & 27,78 & $\begin{array}{l}\text { 1-formyl-2,2,6-trimeth- } \\
\text { yl-3- (3-methyl-but-2-enyl) } \\
\text {-6-cyclohexane }\end{array}$ & 1,05 \\
\hline 21 & 27,94 & $\begin{array}{l}\text { 3-carboxy-4-nitrophenyl } \\
\text { disulfide }\end{array}$ & 0,53 \\
\hline 22 & 28,28 & $\begin{array}{l}((2 \mathrm{E}, 4 \mathrm{E})-3,7,11 \text {-trimeth- } \\
\text { yldodecane-2,4,10-triene }\end{array}$ & 0,34 \\
\hline 23 & 28,33 & $\begin{array}{l}\text { 4,8,13-Cyclotetrade- } \\
\text { catatriene-1,3-diol, } \\
\text { 1,5,9-trimethyl-12- } \\
\text { (1-methylethyl) - }\end{array}$ & 1,37 \\
\hline 24 & 28,45 & cyclopentanone & 1,70 \\
\hline 25 & 28,49 & $\begin{array}{l}\text { Cyclohexanethanol, } \\
\text { 4-methyl- } \beta \text {-methylene- }\end{array}$ & 1,71 \\
\hline 26 & 28,89 & $\begin{array}{l}\text { 2- (2-aminobutane-2-yl-di- } \\
\text { azenyl) -2-methylbuta- } \\
\text { nethanitrile } \\
\end{array}$ & 1,63 \\
\hline 27 & 31,64 & Diisooctylphthalate & 0,68 \\
\hline \multicolumn{3}{|c|}{ Sum } & 100,00 \\
\hline
\end{tabular}

In the results of the analysis of the extract obtained at elevated pressure and temperature, we see a significantly smaller amount of extracted nicotine $(12.29 \%)$, the total number of identified compounds is less, only 28 , which can be explained by partial destruction or by a change in the dissolving ability of carbon dioxide.

Studied on the topic of literature the results indicated there indicate that in some cases the extraction proceeds more fully when the pressure is lowered and this leads to a change in the solubilizing indices of carbon dioxide. We decided to lower the pressure of the extraction process to 120 bar while maintaining the temperature at $60^{\circ} \mathrm{C}$. The data presented in Table 5.

Table 5: Results of chromatography-mass spectrometric analysis of the extract of the plant Nicotiana tabacum I obtained by the method of supercritical fluid $\mathrm{CO}_{2}$ extraction under conditions of 120 bar $60^{\circ} \mathrm{C}$

\begin{tabular}{|c|c|l|c|}
\hline № & $\begin{array}{c}\text { Retention } \\
\text { time, min }\end{array}$ & Name of compounds & $\begin{array}{c}\text { Content } \\
\%\end{array}$ \\
\hline 1 & 6,25 & $\begin{array}{l}\text { 1-butanol, 3-methyl-, } \\
\text { acetate }\end{array}$ & 0,02 \\
\hline 2 & 6,49 & 3-heptanol & 0,01 \\
\hline 3 & 7,03 & Ethanol, 2-butoxy- & 0,17 \\
\hline 4 & 8,53 & Furan, 2-pentyl & 0,01 \\
\hline 5 & 8,83 & Butaneacid, butylether & 0,01 \\
\hline 6 & 9,24 & $\begin{array}{l}\text { Cyclohexanol, 1-meth- } \\
\text { yl-4- (1-methylethenyl) -, } \\
\text { acetate }\end{array}$ & 0,16 \\
\hline 7 & 9,57 & 1-hexanol, 2-ethyl- & 0,01 \\
\hline 8 & 9,89 & Benzaldehyde & 0,01 \\
\hline 9 & 9,99 & Butanoicacid, 4-hydroxy- & 0,01 \\
\hline 10 & 11,77 & Benzylalcohol & 0,03 \\
\hline 11 & 11,98 & Pentanoicacid, 3-methyl- & 0,37 \\
\hline 12 & 12,36 & Benzylacetatedigoxil & 0,14 \\
\hline 13 & 12,91 & hexanoicanhydride & 0,02 \\
\hline 14 & 13,29 & $\begin{array}{l}\text { (R, S) -5-ethyl-6-methyl- } \\
\text { 3E-hepten-2-one }\end{array}$ & 0,04 \\
\hline 15 & 13,54 & Hexanoicacid, anhydride & 0,02 \\
\hline 16 & 13,91 & 4-methyl-5H-furan-2-one & 0,01 \\
\hline 17 & 14,23 & Octanoicacid, ethylester & 0,01 \\
\hline 18 & 14,51 & $\begin{array}{l}\text { 1,1'-bicyclohexyl, 2- } \\
\text { (2-methylpropyl) -, trans- }\end{array}$ & 0,01 \\
\hline 19 & 14,65 & Phenylethylalcohol & 0,06 \\
\hline 20 & 14,99 & 3-Eicozen, (E) - & 0,04 \\
\hline 21 & 15,23 & $\begin{array}{l}\text { 2-Hexadecene, } \\
\text { 3,7,11,15-tetramethyl-, } \\
\text { [R- [R * } \text { R * - (E)]] - }\end{array}$ & 0,03 \\
\hline
\end{tabular}




\begin{tabular}{|c|c|c|c|}
\hline 22 & 15,45 & 1-decanol, 2-methyl- & 0,02 \\
\hline 23 & 15,64 & $\begin{array}{l}\text { 4H-imidazol-4-one, } \\
\text { 2-amino-1,5-dihydro }\end{array}$ & 0,01 \\
\hline 24 & 16,22 & 2-Isopropyl-5-oxohexanal & 0,01 \\
\hline 25 & 16,36 & $\begin{array}{l}\text { Ethanone, 1- (3-methyl- } \\
\text { phenyl) - }\end{array}$ & 0,01 \\
\hline 26 & 17,72 & tetradecane & 0,06 \\
\hline 27 & 18,27 & $\begin{array}{l}\text { 2-buten-1-one, 1- } \\
\text { (2,6,6-trimethyl-1-cyclo- } \\
\text { hexen-1-yl) }\end{array}$ & 0,01 \\
\hline 28 & 19,73 & $\begin{array}{l}\text { 6,8-nonadien-2-one, } \\
\text { 8-methyl-5- (1-methyleth- } \\
\text { yl) -, (E) - }\end{array}$ & 0,31 \\
\hline 29 & 20,02 & $\begin{array}{l}\text { Propane, 2-isocyana- } \\
\text { to-2-methyl- }\end{array}$ & 0,02 \\
\hline 30 & 20,91 & Nicotine & 47,40 \\
\hline 31 & 21,69 & $\begin{array}{l}\text { 7-methoxy-2,2,4,8-te- } \\
\text { tramethyltricyclo [5.3.1.0 } \\
(4.11)] \text { undecane }\end{array}$ & 0,13 \\
\hline 32 & 21,86 & $\begin{array}{l}\text { 5,9-Undecadien-2-one, } \\
\text { 6,10-dimethyl-, (E) - }\end{array}$ & 0,02 \\
\hline 33 & 22,72 & hexadecane & 0,19 \\
\hline 34 & 22,87 & \begin{tabular}{|l|} 
1-dodecanol, \\
3,7,11-trimethyl-
\end{tabular} & 0,01 \\
\hline 35 & 23,14 & Bottledhydroxytoluene & 0,02 \\
\hline 36 & 23,28 & $\begin{array}{l}\text { Phenol, 2,4-bis (1,1-di- } \\
\text { methylethyl) - }\end{array}$ & 0,02 \\
\hline 37 & 23,89 & $\begin{array}{l}\text { Pyridine, 3- (3,4-dihydro- } \\
\text { 2H-pyrrol-5-yl) - }\end{array}$ & 0,24 \\
\hline 38 & 24,15 & $\begin{array}{l}\text { 5-Isopropyl-6-methyl- } \\
\text { hept-3,5-diene-2-ol }\end{array}$ & 1,41 \\
\hline 39 & 24,45 & $\begin{array}{l}\text { Pyridine, 3- (1-meth- } \\
\text { yl-2-pyrrolidinyl) -, N-ox- } \\
\text { ide, (2S) - }\end{array}$ & 0,02 \\
\hline 40 & 24,93 & 1-Heptatriacontanol & 0,03 \\
\hline 41 & 25,02 & heptadecane & 0,03 \\
\hline 42 & 25,36 & Nicotirin & 0,32 \\
\hline 43 & 25,45 & $\begin{array}{l}\text { trans-5-isopropyl-6,7-ep- } \\
\text { oxy-8-hydroxy-8-methyl- } \\
\text { nonan-2-one }\end{array}$ & 0,01 \\
\hline 44 & 25,51 & tert-hexadecanethiol & 0,02 \\
\hline 45 & 25,62 & $\begin{array}{l}\text { Trichloroaceticacid, hexa- } \\
\text { decylether }\end{array}$ & 0,03 \\
\hline 46 & 25,70 & 1-hexadecansulfonyl & 0,02 \\
\hline 47 & 25,81 & octacosanol & 0,02 \\
\hline 48 & 25,94 & 1-trikozen & 0,04 \\
\hline
\end{tabular}

\begin{tabular}{|c|c|c|c|}
\hline 49 & 26,12 & $\begin{array}{l}\text { 5,6,6-trimethyl-5- } \\
\text { (3-oxobut-1-enyl) -1-ox- } \\
\text { aspiro [2.5] octan-4-one }\end{array}$ & 0,16 \\
\hline 50 & 26,62 & $\begin{array}{l}\text { 1-allyl-2-hydroxy-6-meth- } \\
\text { ylcyclohexanecarboxylic } \\
\text { acid }\end{array}$ & 0,07 \\
\hline 51 & 26,88 & 2,3'-dipyridyl & 0,03 \\
\hline 52 & 27,24 & Octadecane & 0,23 \\
\hline 53 & 27,33 & Pentadecanal & 0,14 \\
\hline 54 & 27,59 & $\begin{array}{l}\text { 4- (2,6,6-trimethyl-cyclo- } \\
\text { hexen-1-enyl) butan-2-ol }\end{array}$ & 0,03 \\
\hline 55 & 27,82 & $\begin{array}{l}\text { 6- (3-hydroxy-but-1-enyl) } \\
\text {-1,5,5-trimethyl-7-oxabi- } \\
\text { cyclo [4.1.0] heptan-2-ol }\end{array}$ & 0,03 \\
\hline 56 & 27,93 & $\begin{array}{l}\text { 2,10-dodecadien-1-ol, } \\
\text { 3,7,11-trimethyl-, (Z) - }\end{array}$ & 0,11 \\
\hline 57 & 28,61 & phytol, acetate & 10,87 \\
\hline 58 & 29,05 & Cedran, 8-propoxy- & 0,07 \\
\hline 59 & 29,13 & $\begin{array}{l}\text { Bicyclo [4.4.0] deca-5- } \\
\text { ene-1-acetic acid }\end{array}$ & 0,03 \\
\hline 60 & 29,34 & nonadecan & 0,03 \\
\hline 61 & 29,42 & $\begin{array}{l}\text { 3-buten-2-one, 4- (4-hy- } \\
\text { droxy-2,2,6-trimeth- } \\
\text { yl-7-oxabicyclo [4.1.0] } \\
\text { hept-1-yl) - }\end{array}$ & 0,02 \\
\hline 62 & 29,63 & $\begin{array}{l}\text { 2-pentadecanone, } \\
\text { 6,10,14-trimethyl- }\end{array}$ & 0,10 \\
\hline 63 & 29,81 & $\begin{array}{l}\text { 2-cyclohexen-1-one, } \\
\text { 4- (3-hydroxybutyl) } \\
\text {-3,5,5-trimethyl- }\end{array}$ & 0,22 \\
\hline 64 & 30,03 & $\begin{array}{l}\text { 4,8,13-cyclotetrade- } \\
\text { catatriene-1,3-diol, } \\
\text { 1,5,9-trimethyl-12- } \\
\text { (1-methylethyl) - }\end{array}$ & 0,04 \\
\hline 65 & 30,09 & $\begin{array}{l}\text { 4a, 7,7,10a-tetrameth- } \\
\text { yldodecabenzo [e] } \\
\text { chromen-3-ol }\end{array}$ & 0,05 \\
\hline 66 & 31,09 & Globulol & 0,09 \\
\hline 67 & 31,36 & eicosane & 0,38 \\
\hline 68 & 31,49 & $\begin{array}{l}\text { 2-butanone, 4- } \\
\text { (2,6,6-trimethyl-1-cyclo- } \\
\text { hexen-1-yl) - }\end{array}$ & 0,18 \\
\hline 69 & 31,67 & $\begin{array}{l}\text { 3- (4,8,12-trimethyltride- } \\
\text { cyl) furan }\end{array}$ & 0,08 \\
\hline 70 & 31,78 & $\begin{array}{l}\text { 1,4-bis (1'-hydroxycyclo- } \\
\text { pentyl) -1,3-butadiyne }\end{array}$ & 0,08 \\
\hline 71 & 31,96 & Cotinine & 0,29 \\
\hline 72 & 32,04 & Scraleoxide & 0,10 \\
\hline
\end{tabular}




\begin{tabular}{|c|c|c|c|}
\hline 73 & 32,66 & n-hexadecanoicacid & 0,50 \\
\hline 74 & 32,93 & $\begin{array}{l}\text { Cyclodecacyclotetrade- } \\
\text { cene, } 14,15 \text { - didehydro- } 1 \text {, } \\
4,5,8,9,10,11,12,13,16,17 \\
, 18,19,20 \text {-tetradecahydro }\end{array}$ & 0,09 \\
\hline 75 & 33,06 & $\begin{array}{l}\text { 4a, 7,7,10a-tetrameth- } \\
\text { yl-dodecahydro-benzo [e] } \\
\text { chromen-3-one }\end{array}$ & 0,10 \\
\hline 76 & 33,84 & $\begin{array}{l}\text { 12-Oxatetracyclo [5.2.1.1 } \\
(2,6) .1(8,11)] \text { dodecan- } \\
\text { 3-ol-10-one acetate }\end{array}$ & 0,10 \\
\hline 77 & 33,89 & $\begin{array}{l}\text { Bicyclo [3.1.1] hept-2- } \\
\text { ene, 2,2 '- (1,2-ethanedi- } \\
\text { yl) bis [6,6-dimethyl- }\end{array}$ & 0,12 \\
\hline 78 & 33,95 & $\begin{array}{l}\text { 14-oxatricyclo [9..2.1.0 } \\
(1.10)] \text { tetradecane, } \\
2,6,6,10,11 \text {-pentamethyl- }\end{array}$ & 0,06 \\
\hline 79 & 34,62 & $\begin{array}{l}\text { 1,2-benzenedicarboxylic } \\
\text { acid, butyl-8-methylnonyl } \\
\text { ether }\end{array}$ & 0,15 \\
\hline 80 & 35,06 & sclareolid & 1,42 \\
\hline 81 & 35,15 & Dokosan & 0,22 \\
\hline 82 & 35,57 & $\begin{array}{l}\text { 1H-benzocyclohep- } \\
\text { ten-7-ol, 2,3,4,4a, } \\
\text { 5,6,7,8-octahydro-1,1,4a, } \\
\text { 7-tetramethyl-, cis- }\end{array}$ & 0,21 \\
\hline 83 & 35,90 & Isoaromadendrenepoxide & 0,39 \\
\hline 84 & 36,50 & $\begin{array}{l}\text { 5- (7a-isopropenyl-4,5-di- } \\
\text { methyl-octahydroin- } \\
\text { den-4-yl) -3-methyl-pent- } \\
\text { 2-en-1-ol }\end{array}$ & 2,62 \\
\hline 85 & 36,85 & $\begin{array}{l}\text { 1-penten-3-one, } 1 \text { - } \\
\text { (2,6,6-trimethyl-1-cyclo- } \\
\text { hexen-1-yl) - }\end{array}$ & 2,89 \\
\hline 86 & 37,46 & $\begin{array}{l}\text { 8H-naphtho [1,2-b] } \\
\text { pyran-8-one, 3-eth- } \\
\text { yldodecahydro-3,4a, } \\
\text { 7,7,10a-pentamethyl-, } \\
\text { [3R- }(3 \alpha, 4 a \beta, 6 a \alpha, 10 a \beta, \\
\text { 10ba)] - }\end{array}$ & 0,27 \\
\hline 87 & 37,63 & $\begin{array}{l}\text { 1-naphthalenepropanol, } \\
\alpha \text {-ethenyl decacidro-2-hy- } \\
\text { droxy- } \alpha, 2,5,5,8 \mathrm{a}-\text { pen- } \\
\text { tamethyl, [1R- }\left[1 \alpha\left(\mathrm{R}^{*}\right) \text {, }\right. \\
2 \beta, 4 \mathrm{a} \beta, 8 \mathrm{a \alpha}]]\end{array}$ & 0,29 \\
\hline 88 & 37,90 & $\begin{array}{l}\text { 5- (7a-isopropenyl-4,5-di- } \\
\text { methyl-octahydroin- } \\
\text { den-4-yl) -3-methyl-pen- } \\
\text { ta-2,4-dien-1-ol }\end{array}$ & 0,45 \\
\hline
\end{tabular}

\begin{tabular}{|c|c|c|c|}
\hline 89 & 38,34 & $\begin{array}{l}\text { 2,5,5,8a-tetrameth- } \\
\text { yl-4-methylene-4a, } \\
\text { 5,6,7,8,8a-hexahy- } \\
\text { dro-4H-chromen }\end{array}$ & 3,31 \\
\hline 90 & 38,68 & $\begin{array}{l}\text { 4,8,13-cyclotetrade- } \\
\text { catatriene-1,3-diol, } \\
\text { 1,5,9-trimethyl-12- } \\
\text { (1-methylethyl) - }\end{array}$ & 1,71 \\
\hline 91 & 38,84 & $\begin{array}{l}\text { 1-carbome- } \\
\text { thoxy-1,2,5,5-tetrameth- } \\
\text { yl-cis-decalin (1R, 2S, } \\
\text { 4aS, 8aS) }\end{array}$ & 0,28 \\
\hline 92 & 38,92 & $\begin{array}{l}\text { 4a, 7,7,10a-Tctroameth- } \\
\text { yldodecabenzo [e] } \\
\text { chromen-3-ol }\end{array}$ & 0,23 \\
\hline 93 & 39,08 & isolonigifolmethylester & 0,53 \\
\hline 94 & 39,23 & Hafilin & 0,10 \\
\hline 95 & 39,61 & $\begin{array}{l}\text { 4,8,13-cyclotetrade- } \\
\text { catatriene-1,3-diol, } \\
\text { 1,5,9-trimethyl-12- } \\
\text { (1-methylethyl) - }\end{array}$ & 9,09 \\
\hline 96 & 39,69 & $\begin{array}{l}\text { 5- (1-Isopropenyl-4,5-di- } \\
\text { methylbicyclo [4.3.0] } \\
\text { nonan-5-yl) -3-meth- } \\
\text { yl-2-pentenol acetate }\end{array}$ & 0,86 \\
\hline 97 & 39,93 & $\begin{array}{l}\text { 1-carbome- } \\
\text { thoxy-1,2,5,5-tetrameth- } \\
\text { yl-cis-decalin (1R, 2S, } \\
\text { 4aS, 8aS) }\end{array}$ & 0,85 \\
\hline 98 & 40,24 & Heptacosan & 0,23 \\
\hline 99 & 40,91 & $\begin{array}{l}\text { 5,6,6-trimethyl-5- } \\
\text { (3-oxobut-1-enyl) -1-ox- } \\
\text { aspiro [2.5] octan-4-one }\end{array}$ & 0,17 \\
\hline 100 & 41,23 & $\begin{array}{l}\text { Tetracos-2,6,14,18,22- } \\
\text { pentaene-10,11-diol, } \\
\text { 2,6,10,15,19,23-hexam- } \\
\text { ethyl- }\end{array}$ & 0,10 \\
\hline 101 & 41,55 & Beganalcohol & 0,78 \\
\hline 102 & 41,87 & $\begin{array}{l}\text { 2,5,5,8a-tetrameth- } \\
\text { yl-4-methylene-4a, } \\
\text { 5,6,7,8,8a-hexahy- } \\
\text { dro-4H-chromen }\end{array}$ & 0,26 \\
\hline 103 & 41,98 & $\begin{array}{l}\text { 1-methyl-3- } \\
(2,2,6 \text {-trimethylbicyclo } \\
{[4.1 .0] \text { hept-1-yl) acetic }} \\
\text { acid ester }\end{array}$ & 0,28 \\
\hline 104 & 42,26 & $\begin{array}{l}\text { 4a, 7,7,10a-tetrameth- } \\
\text { yl-dodecahydro-benzo [e] } \\
\text { chromen-3-one }\end{array}$ & 0,39 \\
\hline 105 & 43,04 & 1-Heptatriacontanol & 0,28 \\
\hline
\end{tabular}




\begin{tabular}{|c|c|c|c|}
\hline 106 & 43,55 & $\begin{array}{l}\text { 1H-naphtho [2,1-b] pyran, } \\
\text { 3-ethynyl-dodecahy- } \\
\text { dro-3,4a, 7,7,10a-pen- } \\
\text { tamethyl-, [3R- }(3 \alpha, 4 a \beta, \\
6 a \alpha, 10 a \beta, 10 b a) \text { - }\end{array}$ & 0,22 \\
\hline 107 & 43,82 & $\begin{array}{l}\text { Di (2-propylpentyl) } \\
\text { phthalic acid ester }\end{array}$ & 0,45 \\
\hline 108 & 44,01 & $\begin{array}{l}\text { 2- [4-methyl-6- } \\
\text { (2,6,6-trimethyl- } \\
\text { cyclohex-1-enyl) } \\
\text { hex-1,3,5-trienyl] cyclo- } \\
\text { hexno-1-ene-1-carboxal- } \\
\text { dehyde }\end{array}$ & 0,27 \\
\hline 109 & 44,19 & $\beta$-epi-shiobunol & 0,21 \\
\hline 110 & 44,37 & $\begin{array}{l}\text { (E) -5-isopropyl-8-hy- } \\
\text { droxy-8-methyl-non-6-en- } \\
\text { 2-one }\end{array}$ & 0,23 \\
\hline 111 & 44,53 & $\begin{array}{l}\text { 5- (7a-isopropenyl-4,5-di- } \\
\text { methyl-octahydroin- } \\
\text { den-4-yl) -3-methyl-pen- } \\
\text { ta-2,4-dien-1-ol }\end{array}$ & 0,07 \\
\hline 112 & 45,64 & $\begin{array}{l}\text { Spiro [furan-2 (5H), } 2 \\
\text { '(1'H) -naphto [2,1-b] } \\
\text { furan] -5-one, 3'a, 4', } 5 \\
\text { ', 5'a, 6', 7', 8', 9', 9'a, } \\
\text { 9'b-decahydro-3,3'a, 6', } 6 \\
\text { ', 9'a-pentamethyl-, [2'S- } \\
\text { (2'a, 3'aa, 5 “Ap, 9'aa, } \\
\text { 9'bß)] - }\end{array}$ & 0,70 \\
\hline 113 & 46,15 & $\begin{array}{l}\text { 1-dimethyl (phenyl) sili- } \\
\text { oxydecan }\end{array}$ & 0,14 \\
\hline 114 & 46,40 & Squalene & 0,42 \\
\hline 115 & 47,13 & Koprostan & 0,04 \\
\hline 116 & 47,23 & tetratetracontan & 0,14 \\
\hline 117 & 48,12 & $\begin{array}{l}\text { 5,8,9-endo-10-exo-te- } \\
\text { tramethyltricyclo [6.3.0.0 } \\
(5.11)] \text { undecan-1-car- } \\
\text { boxylic acid }\end{array}$ & 0,25 \\
\hline 118 & 48,57 & $\begin{array}{l}\text { 6,10,14,18,22- tetra- } \\
\text { kozapentaen-2-ol, 3- } \\
\text { bromo-2,6,10, 15,19,23 } \\
\text { - hexamethyl-, (all-E) - }\end{array}$ & 0,13 \\
\hline 119 & 51,30 & 28-Nor-17 $(\mathrm{H})$-gopan & 0,06 \\
\hline 120 & 51,76 & $\begin{array}{l}\text { 2,2,6,6-tetrameth- } \\
\text { yl-4-piperidinyl ester } \\
\text { decanedioic acid }\end{array}$ & 0,85 \\
\hline 121 & 52,06 & DL-a-tocopherol & 0,83 \\
\hline 122 & 52,36 & $\begin{array}{l}\text { 17.a., } 21 \beta-28,30 \text {-bisnor- } \\
\text { fan }\end{array}$ & 0,11 \\
\hline
\end{tabular}

\begin{tabular}{|c|c|l|c|}
\hline 123 & 52,60 & $\begin{array}{l}17-(1,5-d i m e t h y l h e x- \\
\text { yl) -10,13-dimeth- } \\
\text { yl-2,3,4,7, 8,9,10, } \\
11,12,13,14,15,16,17 \\
- \text { tetradecahydro- 1H-cy- } \\
\text { clopenta [ a] phenan- } \\
\text { threne-3-ol }\end{array}$ & 0,15 \\
\hline 124 & 53,74 & Lupan-3-he & 0,06 \\
\hline 125 & 54,67 & stigmasterol & 0,29 \\
\hline \multicolumn{2}{|l|}{ Sum } & 100,00 \\
\hline
\end{tabular}

The data presented in Table 5 indicate that under conditions of supercritical fluid $\mathrm{CO}_{2}$ extraction at a pressure of 120 bar and a temperature of $60^{\circ} \mathrm{C}$, an extremely high yield of nicotine is achieved, which is $47.40 \%$, and this is a very high figure for alkaloids.

Note that in addition to nicotine, its derivatives have been identified: cotinine and nicotirin (Fig.1).<smiles>CN1C(=O)CC[C@H]1c1cccnc1</smiles>

Cotinine<smiles>Cn1cccc1-c1cccnc1</smiles>

Nicotirin

Figure 1: Structural formulas of Cotinine and Nicotirin

In addition, in the extract obtained under these conditions, the largest number of compounds (125) was identified, but almost all of them, except for phytol acetate, are present in minor quantities.

These changes in composition are explained by the fact that in supercritical processes, pressure and temperature are of decisive importance not only on the completeness of extraction, but also on the properties of the extractant [30-34].

For a more detailed study of the influence of the temperature factor on the process of extracting biologically active substances from the aerial part of Nicotiana tabacum I, extraction was carried out at a pressure of 170 bar and a temperature of $80^{\circ} \mathrm{C}$. The results are presented in table 6 . 
Table 6: Results of chromato-mass spectrometric analysis of the extract of the plant Nicotiana tabacum I obtained by the method of supercritical fluid $\mathrm{CO}_{2}$ extraction under conditions of 170 bar $80^{\circ} \mathrm{C}$

\begin{tabular}{|c|c|c|c|}
\hline № & $\begin{array}{l}\text { Retention } \\
\text { time, } \min \end{array}$ & Name of compounds & $\begin{array}{c}\text { Content } \\
\%\end{array}$ \\
\hline 1 & 6,16 & $\begin{array}{l}\text { 1-butanol, 3-methyl-, } \\
\text { acetate }\end{array}$ & 0,03 \\
\hline 2 & 6,97 & Ethanol, 2-butoxy- & 0,29 \\
\hline 3 & 7,22 & Benzene, 1-ethyl-2-methyl- & 0,02 \\
\hline 4 & 7,57 & Buty|AcidHeptylEster & 0,29 \\
\hline 5 & 7,83 & 1,2-propandiol, 3-methoxy- & 1,33 \\
\hline 6 & 8,04 & Pyrazine, 2,6-dimethyl- & 0,10 \\
\hline 7 & 8,31 & 4-cyclopenten-1,3-dione & 0,07 \\
\hline 8 & 8,49 & Furan, 2-pentyl & 0,02 \\
\hline 9 & 8,79 & butanoicacidbutylester & 0,03 \\
\hline 10 & 8,93 & hexanoicacidethylester, & 0,01 \\
\hline 11 & 9,20 & limonene & 0,02 \\
\hline 12 & 9,56 & 1-hexanol, 2-ethyl- & 0,09 \\
\hline 13 & 9,96 & biturolactone & 0,03 \\
\hline 14 & 10,05 & $2(5 \mathrm{H})$-furanone & 0,02 \\
\hline 15 & 11,73 & Benzylalcohol & 0,08 \\
\hline 16 & 14,57 & Sorbicacid & 2,68 \\
\hline 17 & 18,08 & Benzoicacid & 41,68 \\
\hline 18 & 19,25 & $\begin{array}{l}\text { 2H-pyran-2-one, 5,6-dihy- } \\
\text { dro-6-pentyl- }\end{array}$ & 0,19 \\
\hline 19 & 19,45 & $\begin{array}{l}\text { 6,8-nonadien-2-one, } \\
\text { 8-methyl-5- (1-methylethyl) } \\
\text {-, (E) - }\end{array}$ & 0,25 \\
\hline 20 & 19,54 & $\begin{array}{l}\text { Butanoicacid 2,3-dihy- } \\
\text { droxypropyl ester }\end{array}$ & 0,04 \\
\hline 21 & 19,71 & 2-piperidinone & 0,17 \\
\hline 22 & 21,14 & Nicotine & 22,50 \\
\hline 23 & 21,81 & $\begin{array}{l}\text { 7-methoxy-2,2,4,8-te- } \\
\text { tramethyltricyclo [5.3.1.0 } \\
(4.11)] \text { undecane }\end{array}$ & 0,10 \\
\hline 24 & 21,89 & $\begin{array}{l}\text { 5,9-undecadien-2-one, } \\
\text { 6,10-dimethyl- }\end{array}$ & 0,06 \\
\hline 25 & 22,38 & $\begin{array}{l}\text { Bicyclo [3.1.0] hexane-3- } \\
\text { one }\end{array}$ & 0,04 \\
\hline 26 & 22,86 & 7-heptadecene, 1-chloro & 0,05 \\
\hline 27 & 24,13 & $\begin{array}{l}\text { Cyclohexanone, 2,3-di- } \\
\text { methyl-2- (3-oxobutyl) - }\end{array}$ & 0,25 \\
\hline 28 & 24,73 & $\begin{array}{l}2 \text { pyridin-2-ylamino cyclo- } \\
\text { hexyl ester of acetic acid }\end{array}$ & 0,03 \\
\hline
\end{tabular}

\begin{tabular}{|c|c|c|c|}
\hline 29 & 25,45 & Nicotine & 0,06 \\
\hline 30 & 25,74 & $\begin{array}{l}\text { 5,6-dimethyl-2-, benzimi- } \\
\text { dosalion }\end{array}$ & 0,05 \\
\hline 31 & 26,66 & $\begin{array}{l}2(4 \mathrm{H}) \text {-benzofura- } \\
\text { none, 5,6,7,7a-tetrahy- } \\
\text { dro-4,4,7a-trimethyl-, (R) }\end{array}$ & 0,17 \\
\hline 32 & 26,96 & 2,3'-dipyridyl & 0,11 \\
\hline 33 & 27,56 & 3-hydroxy- $\beta$-damascon & 0,13 \\
\hline 34 & 27,69 & Megasigmastiron & 0,42 \\
\hline 35 & 27,83 & $\begin{array}{l}\text { Propyl 2,4-hexadiene } \\
\text { carboxylate }\end{array}$ & 0,55 \\
\hline 36 & 28,40 & $\begin{array}{l}\text { 2-cyclohexen-1-one, } \\
\text { 4- (3-hydroxy-1-butenyl) } \\
\text {-3,5,5-trimethyl- }\end{array}$ & 2,19 \\
\hline 37 & 29,53 & $\begin{array}{l}\text { 1,2-benzenediol, 3,5-bis } \\
\text { (1,1-dimethylethyl) - }\end{array}$ & 0,04 \\
\hline 38 & 30,04 & Farnesylbromide & 0,05 \\
\hline 39 & 30,39 & $\begin{array}{l}\text { Phenol, 2,6-bis (1,1-di- } \\
\text { methylethyl) -4-ethyl- }\end{array}$ & 0,02 \\
\hline 40 & 30,62 & DL-xylitol, 1-benzoate & 1,05 \\
\hline 41 & 31,43 & $\begin{array}{l}\text { Hexadecanoicacid, meth- } \\
\text { ylester }\end{array}$ & 0,19 \\
\hline 42 & 31,66 & $\begin{array}{l}\text { Methyl ester of 3- (1-ace- } \\
\text { tyl-2,2-dimethyl-5-oxocy- } \\
\text { clopentyl) acrylic acid } \\
\end{array}$ & 0,08 \\
\hline 43 & 32,19 & $\begin{array}{l}\text { 5,9,13-pentadecatrien-2- } \\
\text { one, 6,10,14-trimethyl-, } \\
(\mathrm{E}, \mathrm{E}) \text { - }\end{array}$ & 0,23 \\
\hline 44 & 32,34 & $\begin{array}{l}\text { 2-cyclohexen-1-one, 4-hy- } \\
\text { droxy-3,5,5-trimethyl-4- } \\
\text { (3-oxo-1-butenyl) - } \\
\end{array}$ & 0,09 \\
\hline 45 & 32,72 & n-hexadecanoicacid & 1,74 \\
\hline 46 & 33,23 & Bayer 28,589 & 0,04 \\
\hline 47 & 33,89 & $\begin{array}{l}\text { Bicyclo [3.1.1] hept-2-ene, } \\
\text { 2,2'- (1,2-ethanediyl) bis } \\
\text { [6,6-dimethyl- }\end{array}$ & 0,44 \\
\hline 48 & 34,62 & $\begin{array}{l}\text { hept-3-yl phthalic acid } \\
\text { butyl ester, }\end{array}$ & 0,15 \\
\hline 49 & 35,24 & $\begin{array}{l}\text { Cyclopropanebutanoic } \\
\text { acid, } 2 \text { - [[2 - [[2 - [(2-pen- } \\
\text { tylcyclopropyl) methyl] } \\
\text { cyclopropyl] methyl] cyclo- } \\
\text { propyl] methyl] -, methyl } \\
\text { ester }\end{array}$ & 0,09 \\
\hline 50 & 35,61 & $\begin{array}{l}\text { 9,12,15-octadecataric acid, } \\
\text { methyl ester, }(Z, Z, Z) \text { - }\end{array}$ & 0,36 \\
\hline 51 & 35,88 & Resibufagenin & 2,62 \\
\hline 52 & 36,41 & Oleicacid & 0,33 \\
\hline
\end{tabular}




\begin{tabular}{|c|c|c|c|}
\hline 53 & 36,66 & 1-Heptatriacontanol & 1,41 \\
\hline 54 & 36,75 & $\begin{array}{l}\text { Ethyl } 6,9,12,15,18 \text {-genic- } \\
\text { osapentaenoate }\end{array}$ & 0,31 \\
\hline 55 & 37,54 & $\begin{array}{l}\text { Card-20 (22) -enolide, } \\
3,5,14,19 \text {-tetrahydroxy-, } \\
(3 \beta, 5 \beta)\end{array}$ & 0,40 \\
\hline 56 & 38,05 & Epiglobulol & 0,33 \\
\hline 57 & 38,64 & 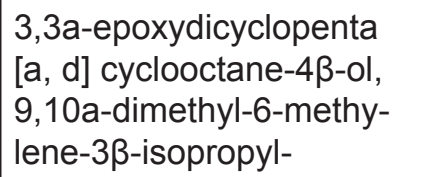 & 1,06 \\
\hline 58 & 39,12 & 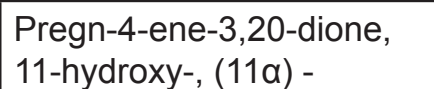 & 0,06 \\
\hline 59 & 39,27 & Dihydroartemisin & 0,12 \\
\hline 60 & 39,46 & $\begin{array}{l}\text { 4,8,13-cyclotetrade- } \\
\text { catatriene-1,3-diol, } \\
\text { 1,5,9-trimethyl-12- } \\
\text { (1-methylethyl) - }\end{array}$ & 4,54 \\
\hline 61 & 39,76 & $\begin{array}{l}\text { 1-naphthalenecarboxylic } \\
\text { acid, 5- (4-carboxy-3- } \\
\text { methyl-3-butenyl) decahy- } \\
\text { dro-1,4a-dimethyl-6-meth- } \\
\text { ylen, [1S- [1a, 4aa, 5a (E), } \\
8 \mathrm{a}] \text { ] - }\end{array}$ & 0,10 \\
\hline 62 & 40,09 & Fluoxymesterone & 0,11 \\
\hline 63 & 40,19 & $\begin{array}{l}\text { 1b, 4a-Epoxy-2H-cy- } \\
\text { clopenta [3,4] cyclo- } \\
\text { prop }[8,9] \text { cycoundec } \\
{[1,2-b] \text { oxyrene- } 5(6 \mathrm{H})} \\
\text {-one, } 7-\text { (acetyloxy) } \\
\text { decahydro-2,9,10- trihy- } \\
\text { droxy-3,6,8,8,10a-pentam- } \\
\text { ethyl }\end{array}$ & 0,23 \\
\hline 64 & 40,62 & $\begin{array}{l}\text { Hexadeca-2,6,10,14-tet- } \\
\text { raen-1-ol, 3,7,11,16-te- } \\
\text { tramethyl- }\end{array}$ & 0,09 \\
\hline 65 & 41,52 & 17-pentatriaconten & 0,21 \\
\hline 66 & 42,20 & $\begin{array}{l}(1 \mathrm{~S}, 2 \mathrm{E}, 4 \mathrm{~S}, 5 \mathrm{R}, 7 \mathrm{E}, 11 \mathrm{E}) \\
\text {-Cembra-2,7,11-triene-4,5- } \\
\text { diol }\end{array}$ & 0,32 \\
\hline 67 & 43,34 & Heptacosan & 0,22 \\
\hline 68 & 43,52 & $\begin{array}{l}\text { 2-hydroxy-1- (hy- } \\
\text { droxymethyl) ethyl ester } \\
\text { hexadecanoic acid }\end{array}$ & 2,60 \\
\hline 69 & 43,81 & $\begin{array}{l}\text { Di (2-propylpentyl) phthalic } \\
\text { acid ester }\end{array}$ & 0,64 \\
\hline 70 & 44,04 & Vidrolhydroxyether & 0,31 \\
\hline
\end{tabular}

\begin{tabular}{|c|c|c|c|}
\hline 71 & 45,59 & $\begin{array}{l}\text { Spiro [furan-2 (5H), } 2 \\
\text { '(1'H) -naphto [2,1-b] furan] } \\
-5-\text {-one, 3'a, 4', 5', 5'a, 6', } \\
\text { 7', 8', 9', 9'a, 9'b-decahy- } \\
\text { dro-3,3'a, 6', 6 ', 9'a-pen- } \\
\text { tamethyl-, [2'S- (2'a, 3'aa, } \\
\text { 5 “Ap, 9'aa, 9'bß)] - }\end{array}$ & 0,15 \\
\hline 72 & 46,22 & Hexacosan & 0,18 \\
\hline 73 & 46,38 & Squalene & 0,23 \\
\hline 74 & 46,65 & $\begin{array}{l}\text { (Z) -, 2-hydroxy-1- (hy- } \\
\text { droxymethyl) ethyl ester of } \\
\text { 9-octadecenoic acid }\end{array}$ & 0,23 \\
\hline 75 & 47,02 & $\begin{array}{l}\text { 2-hydroxy-1- (hy- } \\
\text { droxymethyl) ethyl linole- } \\
\text { nic acid ester (Z, Z, Z) - }\end{array}$ & 0,57 \\
\hline 76 & 47,21 & 2-methyloctacosan & 0,28 \\
\hline 77 & 48,35 & tetratetracontan & 0,22 \\
\hline 78 & 48,93 & tetratriacontan & 0,47 \\
\hline 79 & 49,86 & Triacontane, 1-bromine & 0,26 \\
\hline 80 & 51,47 & tetratetracontan & 0,23 \\
\hline 81 & 52,05 & Vitamin $\mathrm{E}$ & 0,84 \\
\hline 82 & 52,35 & $\begin{array}{l}\text { Phenanthrene, 9-dodec- } \\
\text { yltetradecahydro }\end{array}$ & 0,15 \\
\hline 83 & 52,61 & $\begin{array}{l}\text { 17- (1,5-dimethylhex- } \\
\text { yl) -10,13-dimethyl-2,3, } \\
4,7,8,9,10,11,12, \\
\text { 13,14,15,16,17 -tetradeca- } \\
\text { hydro- 1H-cyclopenta [ a] } \\
\text { phenanthrene-3-ol }\end{array}$ & 0,34 \\
\hline 84 & 54,69 & stigmasterol & 0,78 \\
\hline 85 & 55,74 & $\gamma$-sitosterol & 0,34 \\
\hline \multicolumn{3}{|c|}{ Sun } & 100,00 \\
\hline
\end{tabular}

The content of table 6 indicates that the analysis of the extract of the plant Nicotiana tabacum / by the method of supercritical fluid $\mathrm{CO}_{2}$ extraction under conditions of 170 bar $80^{\circ} \mathrm{C}$ helps to identify a much larger number of compounds (85), to obtain benzoic acid with a high content $(41.68 \%)$. However, the amount of nicotine produced under these conditions is $22.50 \%$. This result for nicotine is comparable to the method of extraction with hexane and extraction at more, which is a factor of thermal destruction of low pressures.

Thus, we can conclude that these conditions are not optimal for the efficient extraction of nicotine. However, the selectivity of the process increases, namely: the dominance nicotine and benzoic acid with a large number of other substances found in minor or trace amounts.

At the end of this phase of the work, an extraction was carried out under a pressure of 120 bar at a temperature of $80^{\circ} \mathrm{C}$ in order to establish the effect of a temperature rise at lower pressures. The data presented in table 7. 
Table 7: Results of chromato-mass spectrometric analysis of the extract of the plant Nicotiana tabacum I obtained by the method of supercritical fluid $\mathrm{CO}_{2}$ extraction under conditions of 120 bar $80^{\circ} \mathrm{C}$

\begin{tabular}{|c|c|c|c|}
\hline № & $\begin{array}{l}\text { Retention } \\
\text { time, min }\end{array}$ & Name of compounds & $\begin{array}{c}\text { Content } \\
\%\end{array}$ \\
\hline 1 & 6,89 & Ethanol, 2-butoxy- & 0,07 \\
\hline 2 & 7,12 & 3-hexene-2-one, 5-methyl- & 0,01 \\
\hline 3 & 8,08 & 2-heptanone, 6-methyl- & 0,01 \\
\hline 4 & 8,76 & Butanoicacidbutylester & 0,01 \\
\hline 5 & 9,17 & $\begin{array}{l}\text { Cyclohexene, 1-methyl-5- } \\
\text { (1-methylethenyl) -, (R) - }\end{array}$ & 0,01 \\
\hline 6 & 9,24 & exo-2-hydroxyenol & 0,01 \\
\hline 7 & 9,39 & 3-methyl butanoicacid & 0,07 \\
\hline 8 & 12,32 & Pentanoicacid, 3-methyl- & 0,26 \\
\hline 9 & 12,91 & Hexanoicacid, anhydride & 0,04 \\
\hline 10 & 13,27 & $\begin{array}{l}\text { (R, S) -5-ethyl-6-methyl- } \\
\text { 3E-hepten-2-one }\end{array}$ & 0,09 \\
\hline 11 & 14,72 & Phenylethylalcohol & 0,05 \\
\hline 12 & 15,00 & $\begin{array}{l}\text { Cyclohexane, 1-eth- } \\
\text { yl-2-propyl }\end{array}$ & 0,02 \\
\hline 13 & 15,23 & 3-Eicozen, (E) - & 0,02 \\
\hline 14 & 15,46 & $\begin{array}{l}\text { 2-Isopropyl-5-meth- } \\
\text { yl-1-heptanol }\end{array}$ & 0,01 \\
\hline 15 & 17,72 & tetradecane & 0,05 \\
\hline 16 & 19,74 & $\begin{array}{l}\text { 6,8-nonadien-2-one, } \\
\text { 8-methyl-5- (1-methylethyl) } \\
\text {-, (E) - } \\
\end{array}$ & 0,21 \\
\hline 17 & 92 & Nicotine & 25,87 \\
\hline 18 & 21,73 & $\begin{array}{l}\text { 7-methoxy-2,2,4,8-te- } \\
\text { tramethyltricyclo [5.3.1.0 } \\
(4.11)] \text { undecane }\end{array}$ & 0,26 \\
\hline 19 & 22,73 & hexadecane & 0,14 \\
\hline 20 & 23,16 & Bottledhydroxytoluene & 0,05 \\
\hline 21 & 23,30 & $\begin{array}{l}\text { Phenol, 2,4-bis (1,1-di- } \\
\text { methylethyl) - } \\
\end{array}$ & 0,03 \\
\hline 22 & 23,93 & $\begin{array}{l}\text { Pyridine, 3- (3,4-dihydro- } \\
\text { 2H-pyrrol-5-yl) - } \\
\end{array}$ & 0,15 \\
\hline 23 & 24,16 & $\begin{array}{l}\text { Cyclohexanone, 2,3-di- } \\
\text { methyl-2- (3-oxobutyl) - }\end{array}$ & 0,94 \\
\hline 24 & 24,50 & nicotine $\mathrm{N}$-oxide & 0,08 \\
\hline 25 & 25,03 & $\begin{array}{l}2(1 \mathrm{H}) \text {-naphthalenone, } \\
3,4,4 \mathrm{a}, 5,6,7,8,8 \mathrm{a} \alpha-\text {-octa- } \\
\text { hydro-5a-hydroxy-4aa, } \\
\text { 7,7-trimethyl-, acetate }\end{array}$ & 0,13 \\
\hline 26 & 25,38 & nicotine & 0,23 \\
\hline
\end{tabular}

\begin{tabular}{|c|c|c|c|}
\hline 27 & 25,69 & 1-hexadecanol, 2-methyl- & 0,06 \\
\hline 28 & 25,93 & 1-dodecanol, 2-octyl- & 0,09 \\
\hline 29 & 26,13 & $\begin{array}{l}\text { 5,6,6-trimethyl-5- } \\
\text { (3-oxobut-1-enyl) -1-oxas- } \\
\text { piro [2.5] octan-4-one }\end{array}$ & 0,12 \\
\hline 30 & 26,32 & 1-genikosyl formate & 0,06 \\
\hline 31 & 26,63 & $\begin{array}{l}\text { 6-ethyl-3-octyl octanoi- } \\
\text { cacid }\end{array}$ & 0,08 \\
\hline 32 & 26,91 & 2,3'-dipyridyl & 0,06 \\
\hline 33 & 27,25 & Octadecane & 0,30 \\
\hline 34 & 27,60 & $\begin{array}{l}\text { Bicyclo [4.3.0] nonane, } \\
\text { 2,2,6,7-tetramethyl-7-hy- } \\
\text { droxy- }\end{array}$ & 0,07 \\
\hline 35 & 27,94 & n-Nonylsuccinicanhydride & 0,19 \\
\hline 36 & 28,60 & Fitol, acetate & 5,85 \\
\hline 37 & 29,06 & $\begin{array}{l}\text { 2-butenal, 2-methyl-4- } \\
\text { (2,6,6-trimethyl-1-cyclo- } \\
\text { hexen-1-yl) - }\end{array}$ & 0,12 \\
\hline 38 & 29,64 & $\begin{array}{l}\text { 2-pentadecanone, } \\
\text { 6,10,14-trimethyl- }\end{array}$ & 0,26 \\
\hline 39 & 29,83 & $\begin{array}{l}\text { 2-cyclohexen-1-one, } \\
\text { 4- (3-hydroxybutyl) } \\
\text {-3,5,5-trimethyl- } \\
\end{array}$ & 0,20 \\
\hline 40 & 30,09 & Isoshiobunon & 0,15 \\
\hline 41 & 30,44 & $\begin{array}{l}\text { 2H-pyran, 2- (7-heptade- } \\
\text { cyloxy) tetrahydro }\end{array}$ & 0,25 \\
\hline 42 & 30,69 & $\begin{array}{l}\text { 5,6,6-trimethyl-5- } \\
\text { (3-oxobut-1-enyl) -1-oxas- } \\
\text { piro [2.5] octan-4-one }\end{array}$ & 0,17 \\
\hline 43 & 31,11 & Epiglobulol & 0,17 \\
\hline 44 & 31,36 & eicosane & 0,26 \\
\hline 45 & 31,52 & 6-epi-shibunol & 0,20 \\
\hline 46 & 31,68 & $\begin{array}{l}\text { 3- (4,8,12-trimethyltridecyl) } \\
\text { furan }\end{array}$ & 0,24 \\
\hline 47 & 31,99 & Continin & 0,34 \\
\hline 48 & 32,47 & $\begin{array}{l}\text { 1-penten-3-one, 1- } \\
\text { (2,6,6-trimethyl-1-cyclo- } \\
\text { hexen-1-yl) - }\end{array}$ & 0,32 \\
\hline 49 & 32,70 & n-hexadecanoicacid & 0,52 \\
\hline 50 & 32,93 & $\begin{array}{l}\text { Cyclodecacyclotetra- } \\
\text { decene, } 14,15 \text { - dide- } \\
\text { hydro-1,4,5, } 8,9,10, \\
11,12,13,16,17,18,19,20 \\
\text { - tetradecahydro }\end{array}$ & 0,12 \\
\hline 51 & 33,88 & $\begin{array}{l}\text { 7H-indeno [5,6-b] furan-7- } \\
\text { one, } 4,4 \mathrm{a}, 5,6,7 \mathrm{a}, 8 \text {-hexa- } \\
\text { hydro }\end{array}$ & 0,43 \\
\hline
\end{tabular}




\begin{tabular}{|c|c|c|c|}
\hline 52 & 34,12 & $\begin{array}{l}\text { 7-methyl-Z-tetrade- } \\
\text { cene-1-ol acetate }\end{array}$ & 0,23 \\
\hline 53 & 34,62 & Dibutylphthalate & 0,39 \\
\hline 54 & 35,07 & sclareolidlactol & 1,54 \\
\hline 55 & 36,52 & $\begin{array}{l}\text { 5- (7a-isopropenyl-4,5-di- } \\
\text { methyl-octagiroinden-4-yl) } \\
\text {-3-methyl-pent-2-en-1-ol }\end{array}$ & 2,67 \\
\hline 56 & 36,85 & $\begin{array}{l}\text { 1-penten-3-one, 1- } \\
\text { (2,6,6-trimethyl-1-cyclo- } \\
\text { hexen-1-yl) - }\end{array}$ & 2,79 \\
\hline 57 & 37,64 & $\begin{array}{l}\text { 1-naphthalenepropanol, } \\
\text { a-ethenyl decacidro-2-hy- } \\
\text { droxy- } \alpha, 2,5,5,8 a-p e n t a m- \\
\text { ethyl, [1R- }\left[1 \alpha\left(R^{*}\right), 2 \beta \text {, }\right. \\
4 a \beta, 8 a \alpha]]\end{array}$ & 1,14 \\
\hline 58 & 38,36 & $\begin{array}{l}\text { 2,5,5,8a-tetrameth- } \\
\text { yl-4-methylene-4a, } \\
\text { 5,6,7,8,8a-hexahy- } \\
\text { dro-4H-chromen }\end{array}$ & 3,11 \\
\hline 59 & 38,86 & $\begin{array}{l}\text { 1-carbome- } \\
\text { thoxy-1,2,5,5-tetrameth- } \\
\text { yl-cis-decalin (1R, 2S, } \\
\text { 4aS, 8aS) }\end{array}$ & 2,71 \\
\hline 60 & 39,10 & $\begin{array}{l}\text { 1-hexene, 2- (p-anisyl) } \\
\text {-5-methyl- }\end{array}$ & 1,27 \\
\hline 61 & 39,66 & \begin{tabular}{|l|} 
4,8,13-cyclotetrade- \\
catatriene-1,3-diol, \\
1,5,9-trimethyl-12- \\
(1-methylethyl) - \\
\end{tabular} & 8,78 \\
\hline 62 & 40,40 & 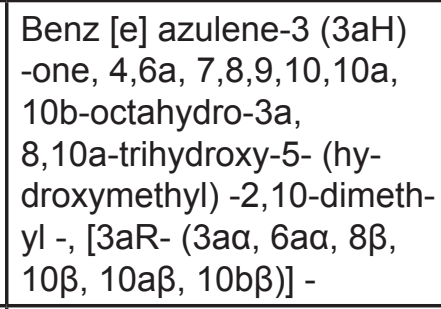 & 0,72 \\
\hline 63 & 40,78 & $\begin{array}{l}\text { 4a, 7,7,10a-tetramethyl- } \\
\text { dodecabenzl [e] chromen- } \\
\text { 3-ol }\end{array}$ & 1,76 \\
\hline 64 & 40,94 & $\begin{array}{l}\text { 5,6,6-trimethyl-5- } \\
\text { (3-oxobut-1-enyl) -1-oxas- } \\
\text { piro [2.5] octan-4-one }\end{array}$ & 1,44 \\
\hline 65 & 41,25 & $\begin{array}{l}\text { 5- (7a-isopropenyl-4,5-di- } \\
\text { methyl-octahydroin- } \\
\text { den-4-yl) -3-methyl-pent- } \\
\text { 2-enal } \\
\end{array}$ & 2,23 \\
\hline 66 & 41,57 & Beganalcohol & 1,87 \\
\hline 67 & 41,99 & $\begin{array}{l}\text { 1-methyl-3- (2,2,6-trimeth- } \\
\text { ylbicyclo [4.1.0] hept-1-yl) } \\
\text { acetic acid ester }\end{array}$ & 2,04 \\
\hline
\end{tabular}

\begin{tabular}{|c|c|c|c|}
\hline 68 & 42,28 & $\begin{array}{l}\text { 4a, 7,7,10a-tetrameth- } \\
\text { yl-dodecahydro-benzo [e] } \\
\text { chromen-3-one }\end{array}$ & 1,05 \\
\hline 69 & 42,60 & Tunbergol & 1,67 \\
\hline 70 & 42,85 & $\begin{array}{l}\text { 4,8,13-cyclotetrade- } \\
\text { catatriene-1,3-diol, } \\
\text { 1,5,9-trimethyl-12- } \\
\text { (1-methylethyl) - }\end{array}$ & 4,03 \\
\hline 71 & 43,60 & $\begin{array}{l}\text { 1H-naphtho [2,1-b] pyran, } \\
\text { 3-ethynyl-dodecahy- } \\
\text { dro-3,4a, 7,7,10a-pentam- } \\
\text { ethyl-, [3R- (3a, 4aß, 6aa, } \\
\text { 10a } \beta, 10 \mathrm{ba}) \text { ] - }\end{array}$ & 1,51 \\
\hline 72 & 43,85 & $\begin{array}{l}\text { Di (2-propylpentyl) phthalic } \\
\text { acid ester }\end{array}$ & 1,14 \\
\hline 73 & 44,23 & 6-epi-shiobunol & 0,65 \\
\hline 74 & 44,40 & $\begin{array}{l}\text { Oxyrandodecanoicacid, } \\
\text { 3-octyl-, cis- }\end{array}$ & 0,95 \\
\hline 75 & 44,57 & $\begin{array}{l}\text { 2- Pentenoic acid, 5- } \\
\text { (decahydro-5,5,8a-trimeth- } \\
\text { yl-2-methylene-1-naphtha- } \\
\text { lenyl) -3-methyl-, methyl } \\
\text { ether, [1R- [1 }(\mathrm{E}), 4 a \beta \text {, } \\
\text { 8aa]] }\end{array}$ & 1,41 \\
\hline 76 & 45,17 & Heptacosan, 1-chloro & 1,25 \\
\hline 77 & 45,43 & $\begin{array}{l}\text { 1-naphthalene penta- } \\
\text { noic acid, decahydro- } \beta \text {, } \\
5,5,8 a-t e t r a m e t h y l-2-\text { meth- } \\
\text { ylene, methyl ether, [1R- } \\
\left.\left[1 \alpha\left(S^{*}\right), 4 a \beta, 8 a \alpha\right]\right]\end{array}$ & 0,68 \\
\hline 78 & 46,17 & 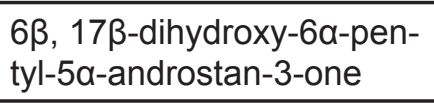 & 0,85 \\
\hline 79 & 46,40 & Squalene & 0,59 \\
\hline 80 & 46,86 & $\begin{array}{l}\text { Spiro [furan-2 (5H), } 2 \\
\text { '(1'H) -naphto [2,1-b] furan] } \\
\text {-5-one, 3'a, 4', 5', 5'a, 6', } \\
7^{\prime}, 8^{\prime}, 9^{\prime}, 9^{\prime} a, 9^{\prime} b-d e c a h y- \\
\text { dro-3,3'a, 6', 6 ', 9'a-pen- } \\
\text { tamethyl-, [2'S- (2'a, 3'aa, } \\
\text { 5 "Ap, 9'aa, 9'b } \beta) \text { ] - }\end{array}$ & 1,12 \\
\hline 81 & 47,70 & $\begin{array}{l}\text { A-nor-cholestan-2-one, } \\
(5 \alpha) \text { - }\end{array}$ & 1,59 \\
\hline 82 & 48,15 & $\begin{array}{l}\text { 5,8,9-endo-10-exo-te- } \\
\text { tramethyltricyclo [6.3.0.0 } \\
(5.11)] \text { undecan-1-carbox- } \\
\text { ylic acid }\end{array}$ & 0,96 \\
\hline 83 & 48,35 & Heptacosan, 1-chloro & 0,38 \\
\hline 84 & 48,58 & $\begin{array}{l}\text { Oxirane, 2,2-dimethyl-3- } \\
(3,7,12,16,20 \text {-pentam- } \\
\text { ethyl-3,7,11,15,19-ge- } \\
\text { nikospentaenyl) }\end{array}$ & 1,20 \\
\hline
\end{tabular}




\begin{tabular}{|c|c|c|c|}
\hline 85 & 51,30 & 28-Nor-17ß (H) -gopan & 0,90 \\
\hline 86 & 51,75 & $\begin{array}{l}\text { Bis }(2,2,6,6 \text {-tetrameth- } \\
\text { yl-4-piperidinyl) decanedi- } \\
\text { oic ester }\end{array}$ & 0,64 \\
\hline 87 & 52,07 & Vitamin $\mathrm{E}$ & 1,00 \\
\hline 88 & 52,36 & 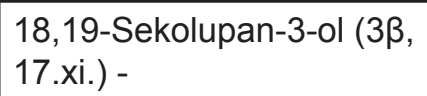 & 0,38 \\
\hline 89 & 52,62 & $\begin{array}{l}\text { 17- (1,5-dimethylhexyl) } \\
\text {-10, 13-dimethyl-2,3,4, } \\
\text { 7,8,9,10,11,12,13,14,15, } \\
\text { 16,17 -tetradecahy- } \\
\text { dro- } 1 \text { H-cyclopenta [ a] } \\
\text { phenanthrene-3-ol }\end{array}$ & 1,06 \\
\hline 90 & 53,74 & Loop-20 (29) -o-28-ol & 0,14 \\
\hline 91 & 54,27 & $\begin{array}{l}\text { 5-cholesten-3-ol, 24-meth- } \\
\text { yl- }\end{array}$ & 0,22 \\
\hline 92 & 54,69 & stigmasterol & 0,35 \\
\hline 93 & 55,74 & $\gamma$-sitosterol & 0,14 \\
\hline \multicolumn{3}{|c|}{ Sum } & 100,00 \\
\hline
\end{tabular}

The data presented in table 7 show that the temperature increase caused a significant decrease in the efficiency of nicotine extraction, compared with the result obtained at 120 bar and $60^{\circ} \mathrm{C}$. In the extract, the nicotine content decreased by $21.53 \%$, that is, almost twice, moreover, the content of phytolacetate fell almost twice, which confirms the pattern in the composition change. Also, despite the temperature increase in the extract, a large number of various compounds have also been identified (93).

\section{CONCLUSION}

In this paper, a comparative analysis of liquid and supercritical extraction of biologically active substances from the plant Nicotiana tabacum I, harvested in the Republic of Kazakhstan, was conducted for the first time.

For the first time, optimal values of pressure and temperature have been established for nicotine release by supercritical fluid extraction with carbon dioxide.

For the first time, the qualitative and quantitative composition of extracts obtained by supercritical fluid extraction with carbon dioxide at various pressures and temperatures has been established.

It should be noted that in all extracts the dominant compound is nicotine, which can be used as a standalone product, or oxidized to nicotinic acid.

Data on the degree of extraction of nicotine with different methods and modes of extraction are presented in Figure 2.

The carried out and analyzed modes of SCF extraction allow us to draw the following conclusions:

- An increase in temperature leads to a change in the properties of the solvent and does not cause ther-

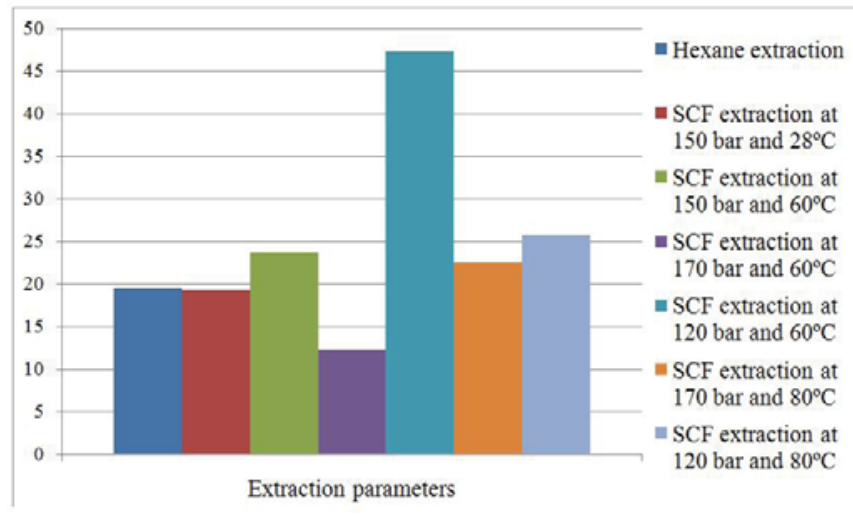

Figure 2: Data on the degree of extraction of nicotine with different methods and modes of extraction

mal degradation processes. This is evidenced by the analysis of the chemical compositions of the obtained extracts: at a relatively high temperature for a BAS(Biologically Active Substances), substances of rather complex composition were identified. The reason for this may be the absence of an oxidizing agent in the system and a short heating time;

- degree of extraction of nicotine at a pressure of 170 bar and a temperature of $80^{\circ} \mathrm{C}$ was comparable with the extraction mode of 150 bar $28^{\circ} \mathrm{C}, 150$ bar $60^{\circ} \mathrm{C}$ and 120 bar $80^{\circ} \mathrm{C}$;

- as a preferred process, we propose a supercritical process with heating up to $60^{\circ} \mathrm{C}$ and a pressure of 120 bar, at which the recovery rate reached $47.40 \%$. In this parameter, it significantly surpasses the classical liquid extraction with organic solvent and the supercritical process without heating.

\section{ACKNOWLEDGMENT}

This work was carried out within the framework of the grant financing program of the SC MES RK BR05236420 "Green Technologies Based on Supercritical Media."

\section{REFERENCES}

1. Herrero, M., Mendiola, J.A., Cifuentes, A., Ibáñez, E.(2010). Supercritical fluid extraction: recent advances and applications. Journal of Chromatography A,vol. 1217 no. 16, 2495-2511doi:10.1016/j. chroma.2009.12.019

2. Diaz, M.S., Brignole, E.A.(2009). Modeling and optimization of supercritical fluid processes. The Journal of Supercritical Fluids, vol. 47, no. 3, 611-618 doi:10.1016/j.supflu.2008.09.006

3. Kassama, L.S., Shi, J., Mittal, G.S.(2008). Optimization of supercritical fluid extraction of lycopene from tomato skin with central composite rotatable design model. Separation and Purification Technology,vol. 60 no. 3, 278-284 doi:10.1016/j.seppur.2007.09.005 
4. Machida, H., Takesue, M., Smith, R.L.(2011). Green chemical processes with supercritical fluids: properties, materials, separations and energy. The Journal of Supercritical Fluids,vol.60, 2-15,doi:10.1016/j. supflu.2011.04.016

5. Qiu S., Sun H., Zhang A.H., Xu H.Y., Yan G.L., Han Y., Wang X.J. (2014) Natural alkaloids: basic aspects, biological roles, and future perspectives. Chin J Nat Med, vol.12, no. 6,401-406,doi:10.1016/ s1875-5364(14)60063-7

6. Ruiz-Rodriguez A., Bronze M.R., Ponte M.N. (2008) Supercritical fluid extraction of tobacco leaves: A preliminary study on the extraction of solanesol. J. Supercrit. Flu, vol.45, no.2,171-176,doi:10.1016/j. supflu.2007.10.011

7. Myers R. L. (2007) The Most Important Chemical Compounds: A Reference Giude Greenwood Press.

8. Schraufnagel D. E., Blasi F., David C. L., Mark J., Raul S.; Richard Van Zyl-Smit(2014) Electronic Cigarettes. A Position Statement of the Forum of International Respiratory Societies. American Journal of Respiratory and Critical Care Medicine,vol.190 no. 6,611-618,doi:10.1164/rccm.201407-1198pp

9. Bruin J. E., Gerstein H. C., Holloway A. C. (2010) Long-term consequences of fetal and neonatal nicotine exposure: a critical review. Toxicological Sciences, vol.116,no. 2,364-374,doi:10.1093/toxsci/ kfq103

10. Blankenship J.D., Houseknecht J.B., Pal S., Bush L.P., Grossman R.B., Schardl C.L. (2005) Biosynthetic precursors of fungal pyrrolizidines, the loline alkaloids. Chembiochem, vol.6, no. 6,10161022,doi:10.1002/cbic.200400327

11. Cushnie T.P., Cushnie B., Lamb A.J. (2014) Alkaloids: An overview of their antibacterial, antibiotic-enhancing and antivirulence activities.Int J Antimicrob Agents,vol .44, no. 5, 377-386, doi:10.1016/j.jjantimicag.2014.06.001

12. Caprioli G., Sagratini G., Vittori S. (2018) Optimization of an extraction procedure for the simultaneous quantification of riboflavin, nicotinamide and nicotinic acid in anchovies (Engraulisenrasicolus) by high-performance liquid chromatography-tandem mass spectrometry .J. Food Comp. Anal, vol. 66, 2329, doi:10.1016/j.jfca.2017.11.004

13. Mahpishanian S., Sereshti H. Graphene. (2014) oxide-based dispersive micro-solid phase extraction for separation and preconcentration of nicotine from biological and environmental water samples followed by gas chromatography-flame ionization detection.Talanta, vol.130, 71-77, doi:10.1016/j.talanta.2014.06.004

14. Anastas P., Eghbali N. (2010). Green chemistry: principles and practice. Chem. Soc,vol. 39, no. 1, 301-312, doi:10.1039/b918763b
15. Azmir J., Zaidul I.S.M., Rahman M.M., Sharif, K.M., Mohamed, A., Sahena, F., Jahurul, M.H.A., Ghafoor, K., Norulaini, N.A.N., Omar, A.K.M.(2013). Techniques for extraction of bioactive compounds from plant materials: a review. J. Food Eng,vol. 117, no. 4, 426-436, sci-hub.tw/10.1016/j.jfoodeng.2013.01.014

16. Barba F.J., Zhu Z., Koubaa M., Sant'Ana A.S., Orlien V.(2016). Green alternative methods for the extraction of antioxidant bioactive compounds from winery wastes and by-products: a review. Trends Food Sci. Technol,vol. 49, 96-109, doi:10.1016/j. tifs.2016.01.006

17. Bernardo-Gil, G., Oneto, C., Antunes, P., Rodriguez, M.F., Empis J.M.(2001).Extraction of lipids from cherry seed oil using supercritical carbon dioxide. Eur. Food Res.Tech,vol. 212, no. 2, 170-174, doi:10.1007/s002170000228

18. Bernhoft, A.(2010).A brief review on bioactive compounds in plants. In: Bernhoft, A. (Eds.), Bioactive compounds in plants: benefits and risks for man and animals. Novus Forlag, Norway

19. Brunner, G., (2005). Supercritical fluids: technology and application to food processing. J. Food Eng,vol. 67, no. 1-2, 21-33, doi:10.1016/j.jfoodeng.2004.05.060

20. Cavero, S., García-Risco, M.R., Marín, F.R., Jaime, L., Santoyo, S., Senorans, F.J, Reglero, G., Ibáñez, E.(2006). Supercritical fluid extraction of antioxidant compounds from oregano. Chemical and functional characterization via LC-MS and in vitro assays. J. Supercrit. Fluids,vol. 38, no. 1, 62-69, doi:10.1016/j. supflu.2005.01.003

21. Chemat, F., Vian, M.A., Cravotto G. (2012). Green extraction of natural products: concept and principles. Int. J. Molec. Sci,vol.13, no. 7, 8615-8627, doi:10.3390/ijms13078615

22. Daood, H.G., Illes, V., Gnayfeed, M.H., Meszaros, B., Horvath, G., Biacs, P.A. (2002). Extraction of pungent spice paprika by supercritical carbon dioxide and subcritical propane, J. Supercrit.Fluids.Vol. 23, no. 2, 143-152, doi:10.1016/s0896-8446(02)00022-0

23. De Lucas, A., Rincon, J., Gracia, I. (2003). Influence of operation variables on quality parameters of olive husk oil extracted with CO2: Three-step sequential extraction. J. Am. Oil Chem. Soc. Vol. 80, no. 2, 181188, doi:10.1007/s11746-003-0674-4

24. Fernández, M.D.L.Á., Espino, M., Gomez, F.J. V, Silva, M.F.(2018). Novel approaches mediated by tailor-made green solvents for the extraction of phenolic compounds from agro-food industrial by-products. Food Chem. vol. 239, 671-678, doi:10.1016/j. foodchem.2017.06.150 
25. Gámiz-Gracia, M., Luque de Castro, M.D.(2000). Continuous subcritical water extraction of medicinal plant essential oil: comparison with conventional techniques. Talanta, vol. 51, no. 6, 1179-1185, doi:10.1016/s0039-9140(00)00294-0

26. Gil-Chávez, J. G., Villa, J.A., Fernando Ayala-Zavala, J., Basilio Heredia, J., Sepulveda, D., Yahia, E.M., González-Aguilar, G.A.(2013). Technologies for extraction and production of bioactive compounds to be used as nutraceuticals and food ingredients: an overview.Compr. Rev. Food Sci. Food Saf, vol. 12, no. 1, 5-23, doi:10.1111/1541-4337.12005

27. Han, X., Cheng, L., Zhang, R., Bi, J.(2009).Extraction of safflower seed oil by supercritical CO2. J. Food Eng,vol.92, no. 4, 370-376, doi:10.1016/j. jfoodeng.2008.12.002

28. Herrero, M., Castro-Puyana, M., Mendiola, J.A., Ibanez, E.(2013).Compressed fluids for the extraction of bioactive compounds. Trends Anal. Chem,vol. 43, 67-83, doi:10.1016/j.trac.2012.12.008

29. Herrero, M., Cifuentes, A., Ibanez, E.(2006). Suband supercritical fluid extraction of functional ingredients from different natural sources: Plants, foodby-products, algae and microalgae: A review. Food Chem, vol. 98, no. 1, 136-148, doi:10.1016/j.foodchem.2005.05.058

30. Jerković, I., Molnar, M., Vidović, S., Vladić, J., Jokić, S.(2017). Supercritical CO2 Extraction of Lavandulaangustifolia Mill.flowers: Optimization of Oxygenated Monoterpenes, Coumarin and Herniarin Content. Phytochem. Anal,vol. 28, no. 6,558-566, DOI: 10.1002/pca.2705
31. Jokić, S., Bijuk, M., Aladić, K., Bilić, M., Molnar, M.(2016).Optimization of supercritical CO2 extraction of grape seed oil using response surface methodology. Int. J. Food Sci. Tech, vol.51, no. 2, 403-410, doi:10.1111/ijfs. 12986

32. Jokić, S., Jerković, I., Rajić, M., Aladić, K., Bilić, M., Vidović, S. (2017). SC-CO2 extraction of Vitexagnus-castus L. fruits: the influence of pressure, temperature and water presoaking on the yield and GC-MS profiles of the extracts in comparison to the essential oil composition. J. Supercrit. Fluids,vol.123, 50-57, doi:10.1016/j.supflu.2016.12.007

33. Kotnik, P., Škerget, M., Knez, Ž.(2006).Kinetics of supercritical carbon dioxide extraction of borage and evening primrose seed oil. Eur. J. Lipid Sci. Technol,vol.108, no. 7, 569-576, doi:10.1002/ ejlt.200600070

34. Cvjetko B., Vidovic S., Radojcil.' c, Joki S.(2018). New perspective in extraction of plant biologically active compounds by green solvents. Food and Bioproducts Processing,vol. 109, 52-73, doi. org/10.1016/j.fbp.2018.03.001 